\title{
Cooling force on ions in a magnetized electron plasma
}

\author{
Hrachya B. Nersisyan ${ }^{1, *}$ and Günter Zwicknagel ${ }^{2, \dagger}$ \\ ${ }^{1}$ Plasma Theory Group, Institute of Radiophysics and Electronics, 0203 Ashtarak, Armenia \\ ${ }^{2}$ Institut für Theoretische Physik II, Universität Erlangen-Nürnberg, Staudtstraße 7, D-91058 Erlangen, Germany
}

(Received 3 March 2013; published 9 July 2013)

\begin{abstract}
Electron cooling is a well-established method to improve the phase space quality of ion beams in storage rings. In the common rest frame of the ion and the electron beam, the ion is subjected to a drag force and it experiences a loss or a gain of energy which eventually reduces the energy spread of the ion beam. A calculation of this process is complicated as the electron velocity distribution is anisotropic and the cooling process takes place in a magnetic field which guides the electrons. In this paper the cooling force is calculated in a model of binary collisions (BC) between ions and magnetized electrons, in which the Coulomb interaction is treated up to second order as a perturbation to the helical motion of the electrons. The calculations are done with the help of an improved BC theory which is uniformly valid for any strength of the magnetic field and where the second-order two-body forces are treated in the interaction in Fourier space without specifying the interaction potential. The cooling force is explicitly calculated for a regularized and screened potential which is both of finite range and less singular than the Coulomb interaction at the origin. Closed expressions are derived for monochromatic electron beams, which are folded with the velocity distributions of the electrons and ions. The resulting cooling force is evaluated for anisotropic Maxwell velocity distributions of the electrons and ions.
\end{abstract}

DOI: 10.1103/PhysRevSTAB.16.074201

\section{INTRODUCTION}

In most experiments with particle beams a high phase space density is desired. In electron cooling of ion beams [1] this is achieved by mixing the ion beam with a comoving electron beam which has a very small longitudinal momentum spread. In the rest frame of the beams the cooling process may be viewed as the stopping of ions in an electron plasma [2-5]. More recently, electron cooling has also been used in traps for precision experiments like CPT tests with antihydrogen [6,7] or planned QED tests with highly charged ions in HITRAP [8]. In these applications the presence of strong external magnetic fields constitutes a theoretical challenge [9], as its influence on the cooling which the magnetized electrons exert on the ions (antiprotons) is not so obvious as earlier models might suggest. In the dielectric theory the drag on the ion is due to the polarization it creates in its wake. This can be either calculated in linear response (LR) $[10,11]$ or numerically by a particle-in-cell (PIC) simulation of the underlying nonlinear Vlasov-Poisson equation [12,13]. While the LR requires cutoffs to exclude hard collisions of close particles the collectivity of the excitation can be

\footnotetext{
*hrachya@irphe.am

† guenter.zwicknagel@physik.uni-erlangen.de
}

Published by the American Physical Society under the terms of the Creative Commons Attribution 3.0 License. Further distribution of this work must maintain attribution to the author(s) and the published article's title, journal citation, and DOI.
PACS numbers: 52.20.Hv, 34.50.Bw, 52.40.Mj, 29.27.Bd

taken into account in both approaches. In the complementary binary collision (BC) approximation the drag force is accumulated from the velocity transfers in individual collisions. This has been calculated by scattering statistical ensembles of magnetized electrons from the ions in the classical trajectory Monte Carlo method (CTMC) [13-18], and by treating the Coulomb interaction as a perturbation to the helical motion of the electrons [19-25]. The observed cooling force $\mathbf{F}\left(\mathbf{v}_{i}\right)$ on an individual ion is obtained by integrating with respect to the impact parameter and the electrons velocity distribution. The ion velocity $\mathbf{v}_{i}$ is measured with respect to the center of that distribution. As in electron cooler the electrons are accelerated from the cathode, their velocity distribution is flattened longitudinally, but the spread does not vanish. Since the cooling force on slow ions and therefore the cooling process depends critically on the details of the velocity distribution, a treatment employing a realistic velocity distribution is desirable.

The purpose of this paper is the application of a secondorder perturbative $\mathrm{BC}$ model for calculating the magnetized cooling force on a uniformly moving individual heavy ion as well as on a heavy ion beam. In previous approaches $[19,20]$ three regimes are identified, depending on the relative size of the cyclotron radius, the distance of the closest approach, and the pitch of the helix. The present paper is based on our earlier studies in Refs. [21-25] where the second-order energy transfers for individual collisions of electron-ion [21-24], of any two identical particles, like e.g. electron-electron [24] and finally of two gyrating 
arbitrary charged particles [25] have been calculated with the help of an improved BC treatment. This treatment is - e.g. unlike Refs. $[19,20]$ — valid for any strength of the magnetic field. In Sec. II we introduce a perturbative binary collision formulation in terms of the binary force acting between an ion and a magnetized electron, and derive general expressions for the second-order (with respect to the interaction potential) cooling forces. In contrast to the previous investigations in Refs. [21-25], we here consider the (macroscopic) cooling forces which are obtained by integrating the binary force of an individual electron-ion interaction with respect to the impact parameter and the velocity distribution function of electrons. That is, the cooling force for monoenergetic electrons is folded with an anisotropic velocity distribution which is typical for electron cooling of ion beams in storage rings, where the velocity spread is much smaller longitudinal than transverse to the guiding magnetic field. The resulting expressions involve all cyclotron harmonics of the electrons' helical motion, and are valid for any interaction potential and any strength of the magnetic field and anisotropy of the velocity distribution of the electron beam. In Sec. IIC we present explicit analytic expressions of this second-order cooling force for the specific case of a regularized and screened interaction potential [26,27] which is both of finite range and less singular than the Coulomb interaction at the origin and which includes as limiting cases the Debye (i.e., screened) and the Coulomb potentials. For comparison of our expressions with previous approaches we consider in Sec. III the corresponding asymptotic expressions for large and small ion velocities and strong and vanishing magnetic fields. The analytical expressions presented in Sec. IIC are evaluated numerically in Sec. IV using parameters of the ESR storage ring at GSI [28-30]. In particular, we compare our approach with the CTMC simulations and the empirical formula of Parkhomchuk [31,32]. In Sec. V we calculate the magnetized cooling force averaged with respect to the ion beam velocity distribution function. As in Sec. II C a similar anisotropic distribution is used for averaging with respect to the ion velocity distribution. Furthermore, in Sec. VA for the resulting cooling force the asymptotic expressions for large and small ion velocities and strong and vanishing magnetic fields are given. In Sec. V B we compare our approach with the experimental data of the ESR storage ring [28-30]. The results are summarized and discussed in Sec. VI. In Appendix A we compare our asymptotic expressions for the cooling force with those obtained in Ref. [33] and demonstrate that the deviations between both treatments are related to the divergent nature of the bare Coulomb interaction employed in Ref. [33]. The regularization parameter and the screening length involved in the interaction potential are specified and discussed in Appendices B and C.

\section{THEORETICAL MODEL}

\section{A. Binary collision (BC) formulation}

We consider two point charges with masses $m, M$ and charges $-e, Z e$, respectively, moving in a homogeneous magnetic field $\mathbf{B}=B \mathbf{b}$. We assume that the particles interact with the potential $-Z k^{2} U(\mathbf{r})$ with $k^{2}=$ $e^{2} / 4 \pi \varepsilon_{0}$, where $\varepsilon_{0}$ is the permittivity of the vacuum and $\mathbf{r}=\mathbf{r}_{1}-\mathbf{r}_{2}$ is the relative coordinate of the colliding particles. For two isolated charged particles this interaction is given by the Coulomb potential, i.e. $U_{\mathrm{C}}(\mathbf{r})=1 / r$. In plasma applications $U_{\mathrm{C}}$ is modified by many-body effects and the related screening and turns into an effective interaction. In general, this effective interaction, which is related to the wakefield induced by a moving ion, is nonspherically symmetric and depends also on the ion velocity. For any BC treatment, however, this complicated ion-plasma interaction must be approximated by an effective two-particle interaction $U(\mathbf{r})$. This effective interaction $U$ may be modeled by a spherically symmetric Debye-like screened interaction $U_{\mathrm{D}}(\mathbf{r})=e^{-r / \lambda} / r$ with a screening length $\lambda$, given e.g. by the Debye screening length $\lambda_{\mathrm{D}}$, see, for example [34], in case of low ion velocities and an effective velocity-dependent screening length $\lambda\left(v_{i}\right)$ for larger ion velocities $v_{i}$, see [35-37]. Further details on the choice of the effective interaction $U(\mathbf{r})$ are given in Appendix B. To cure problems related to the Coulomb singularity in a classical picture and preventing particles (for $Z>0$ ) from falling into the center of these potentials, the screened interaction $U_{\mathrm{D}}$ is replaced with an effective interaction $U_{\mathrm{R}}$ which is regularized at the origin, taking for example $U_{\mathrm{R}}(\mathbf{r})=$ $\left(1-e^{-r / \lambda}\right) e^{-r / \lambda} / r[26,27]$. Here the use of this regularized interaction essentially represents an alternative implementation of the standard (lower) cutoff procedure needed to handle the hard collisions in a classical perturbative approach. Hence, we consider $\lambda$ as a given constant or as a function of the classical collision diameter (see Appendix B).

In the presence of an external magnetic field, the Lagrangian and the corresponding equations of particles motion cannot, in general, be separated into parts describing the relative motion and the motion of the center of mass (cm) [23]. However, in the case of heavy ions, i.e. $M \gg m$, the equations of motion can be simplified by treating the $\mathrm{cm}$ velocity $\mathbf{v}_{\mathrm{cm}}$ as a constant and equal to the ion velocity $\mathbf{v}_{i}$, i.e. $\mathbf{v}_{\mathrm{cm}}=\mathbf{v}_{i}=$ const. Then the equation of relative motion turns into

$$
\dot{\mathbf{v}}(t)+\omega_{c}[\mathbf{v}(t) \times \mathbf{b}]=-\omega_{c}\left[\mathbf{v}_{i} \times \mathbf{b}\right]-\frac{Z \ell^{2}}{m} \mathbf{f}[\mathbf{r}(t)],
$$

where $\mathbf{v}(t)=\dot{\mathbf{r}}(t)=\mathbf{v}_{e}(t)-\mathbf{v}_{i}$ is the relative electron-ion velocity, $-Z k^{2} \mathbf{f}[\mathbf{r}(t)](\mathbf{f}=-\partial U / \partial \mathbf{r})$ is the force exerted by the ion on the electron, and $\omega_{c}=e B / m$ is the electron cyclotron frequency. 
It is now useful to introduce the velocity correction through relations $\delta \mathbf{v}(t)=\mathbf{v}_{e}(t)-\mathbf{v}_{e 0}(t)=\mathbf{v}(t)-\mathbf{v}_{0}(t)$, where $\mathbf{v}_{e 0}(t)$ and $\mathbf{v}_{0}(t)$ are the unperturbed electron and relative velocities, respectively, with $\mathbf{v}_{0}(t)=\dot{\mathbf{r}}_{0}(t)=$ $\mathbf{v}_{e 0}(t)-\mathbf{v}_{i}$,

$$
\begin{gathered}
\mathbf{r}_{0}(t)=\mathbf{R}_{0}+\mathbf{v}_{r} t+a\left[\mathbf{u} \sin \left(\omega_{c} t\right)-[\mathbf{b} \times \mathbf{u}] \cos \left(\omega_{c} t\right)\right], \\
\delta \dot{\mathbf{v}}(t)+\omega_{c}[\delta \mathbf{v}(t) \times \mathbf{b}]=-\frac{Z k^{2}}{m} \mathbf{f}[\mathbf{r}(t)]
\end{gathered}
$$

and $\delta \mathbf{v}(t) \rightarrow 0$ at $t \rightarrow-\infty$. In Eq. (2) $\mathbf{u}=(\cos \varphi, \sin \varphi)$ is the unit vector perpendicular to the magnetic field, the angle $\varphi$ is the initial phase of the electron's helical motion, $v_{e \|}$ and $v_{e \perp}$ (with $v_{e \perp} \geq 0$ ) are the unperturbed components of the electron velocity parallel and perpendicular to $\mathbf{b}$, respectively, $\mathbf{v}_{r}=v_{e \|} \mathbf{b}-\mathbf{v}_{i}$ is the relative velocity of the guiding center of the electrons, and $a=v_{e \perp} / \omega_{c}$ is the cyclotron radius. In Eq. (2), the variables $\mathbf{u}$ and $\mathbf{R}_{0}$ are independent and are defined by the initial conditions. In Eq. (3) $\mathbf{r}(t)=\mathbf{r}_{e}(t)-\mathbf{v}_{i} t$ is the ion-electron relative coordinate. We also introduce the variable $\mathbf{s}=\mathbf{R}_{0 \perp}=$ $\mathbf{R}_{0}-\mathbf{n}_{r}\left(\mathbf{n}_{r} \cdot \mathbf{R}_{0}\right)$ which is the component of $\mathbf{R}_{0}$ perpendicular to the relative velocity vector $\mathbf{v}_{r}$ with $\mathbf{n}_{r}=\mathbf{v}_{r} / v_{r}$. From Eq. (2) we can see that $\mathbf{s}$ is the distance of closest approach between the ion and the guiding center of the electron's helical motion.

We seek an approximate solution of Eq. (3) in which the interaction force between the ion and electrons is considered as a perturbation. Thus we are looking for a solution of Eq. (3) for the variables $\mathbf{r}$ and $\mathbf{v}$ in a perturbative manner $\mathbf{r}=\mathbf{r}_{0}+\mathbf{r}_{1}+\cdots, \mathbf{v}=\mathbf{v}_{0}+\mathbf{v}_{1}+\cdots$, where $\mathbf{r}_{0}(t), \mathbf{v}_{0}(t)$ are the unperturbed ion-electron relative coordinate and velocity, respectively, $\mathbf{r}_{n}(t), \mathbf{v}_{n}(t)(n=1,2, \ldots)$ are the $n$th order perturbations of $\mathbf{r}(t)$ and $\mathbf{v}(t)$, which are proportional to $Z^{n}$.

The parameter of smallness which justifies such kind of expansion can be read off from a dimensionless form of the equation of motion [Eq. (3)] by scaling lengths in units of the screening length $\lambda$, velocities in units of the initial relative velocity $v_{0}$, and time in units of $\lambda / v_{0}$. In terms of the scaled quantities, $\tilde{\mathbf{r}}, \delta \tilde{\mathbf{v}}, \tilde{\mathbf{f}}=\lambda^{2} \mathbf{f}$, and $\tilde{\omega}_{c}=\omega_{c} \lambda / \boldsymbol{v}_{0}$, Eq. (3) turns into

$$
\delta \dot{\tilde{\mathbf{v}}}(t)+\tilde{\omega}_{c}[\delta \tilde{\mathbf{v}}(t) \times \mathbf{b}]=-\frac{Z k^{2}}{m v_{0}^{2} \lambda} \tilde{\mathbf{f}}[\tilde{\mathbf{r}}(t)] .
$$

A perturbative treatment is essentially applicable in cases where $|Z| k^{2} / m v_{0}^{2} \lambda<1$, that is, when the (initial) kinetic energy of relative motion $m v_{0}^{2} / 2$ is large compared to the characteristic potential energy $|Z| e^{2} / \lambda$ in a screened Coulomb potential. Or expressed in velocities, the initial relative velocity $v_{0}$ must exceed the characteristic velocity $v_{d}=\left(|Z| e^{2} / m \lambda\right)^{1 / 2}$, that is, $v_{d}$ here demarcates the perturbative from the nonperturbative regime. If this condition is met not only for a single ion-electron collision but in the average over the electron distribution, e.g. by replacing $v_{0}$ with the averaged initial ion-electron relative velocity $\left\langle v_{0}\right\rangle$, i.e.

$$
\left\langle v_{0}\right\rangle \gtrsim v_{d}=\left(\frac{|Z| e^{2}}{m \lambda}\right)^{1 / 2}
$$

we are in a regime of weak ion target, or here, weak ionelectron coupling, which allows the use of perturbative treatments [besides BC also e.g. linear-response (LR)]. For nonmagnetized electrons this is discussed in much detail in Refs. [35,36]. Even though the particle trajectories are much more intricate in the presence of an external magnetic field, the given definitions and demarcations of coupling regimes are basically the same for magnetized electrons. That is, the applicability of a perturbative treatment is essentially related to the charge state $Z$ of the ion and the typical range $\lambda$ of the effective interaction, but not directly on the strength $B$ of the magnetic field. The latter may affect the critical velocity $v_{d}$ only implicitly via a possible change of the effective screening length $\lambda$ with $B$.

The equation for the first-order velocity correction is obtained from Eq. (3) replacing on the right-hand side the exact relative coordinate $\mathbf{r}(t)$ by $\mathbf{r}_{0}(t)$ with the solutions $\mathbf{v}_{1}(t)=\dot{\mathbf{r}}_{1}(t)$ and

$$
\begin{aligned}
\mathbf{r}_{1}(t)= & \frac{Z k^{2}}{m}\left(-\mathbf{b} \mathcal{Q}_{\|}(t)+\operatorname{Re}\left\{\mathbf{b}\left[\mathbf{b} \cdot \mathcal{Q}_{\perp}(t)\right]\right.\right. \\
& \left.\left.-\mathcal{Q}_{\perp}(t)+i\left[\mathbf{b} \times \mathcal{Q}_{\perp}(t)\right]\right\}\right) .
\end{aligned}
$$

Here we have introduced the following abbreviations:

$$
\begin{aligned}
& \mathcal{Q}_{\|}(t)=\int_{-\infty}^{t} \mathbf{b} \cdot \mathbf{f}\left[\mathbf{r}_{0}(\tau)\right](t-\tau) d \tau \\
& \mathcal{Q}_{\perp}(t)=\frac{1}{i \omega_{c}} \int_{-\infty}^{t} \mathbf{f}\left[\mathbf{r}_{0}(\tau)\right]\left[e^{i \omega_{c}(t-\tau)}-1\right] d \tau
\end{aligned}
$$

and have assumed that all corrections vanish at $t \rightarrow-\infty$. As will be shown below, Eqs. (2) and (6) completely determine the second-order cooling force on the ion.

\section{B. Second-order cooling forces}

We now consider the interaction process of an individual ion with a homogeneous electron beam described by a velocity distribution function $f\left(\mathbf{v}_{e}\right)$ and a density $n_{e}$. We assume that the ion experiences independent binary collisions (BCs) with the electrons. The total cooling force acting on the ion is then obtained by multiplying the binary force $Z k^{2} \mathbf{f}[\mathbf{r}(t)]$ by the element of the electron relative flux $n_{e} v_{r} d^{2} \mathbf{s} d t$ (where $\mathbf{s}$ is the impact parameter introduced above which is perpendicular to the relative velocity $\mathbf{v}_{r}$ ) and integrating with respect to time and folding with velocity distribution of the electrons. The result reads

$$
\mathbf{F}\left(\mathbf{v}_{i}\right)=Z k^{2} n_{e} \int d \mathbf{v}_{e} f\left(\mathbf{v}_{e}\right) v_{r} \int d^{2} \mathbf{s} \int_{-\infty}^{\infty} \mathbf{f}[\mathbf{r}(t)] d t
$$

and is an exact relation for uncorrelated BCs of the ion with electrons. We evaluate this expression within a systematic 
perturbative treatment. First, we introduce the two-particle interaction potential $U(\mathbf{r})$ and the binary force $\mathbf{f}(\mathbf{r})$ is written using Fourier transformation in space. Furthermore, the factor $e^{i \mathbf{k} \cdot \mathbf{r}(t)}$ in the Fourier transformed binary force is expanded in a perturbative manner as $e^{i \mathbf{k} \cdot \mathbf{r}(t)} \simeq e^{i \mathbf{k} \cdot \mathbf{r}_{0}(t)}\left\{1+i\left[\mathbf{k} \cdot \mathbf{r}_{1}(t)\right]\right\}$, where $\mathbf{r}_{0}(t)$ and $\mathbf{r}_{1}(t)$ are the unperturbed and the first-order corrected relative coordinates, Eqs. (2) and (6), respectively. Thus the binary force within second-order perturbative treatment turns into

$$
\begin{aligned}
\mathbf{f}[\mathbf{r}(t)] & =-i \int d \mathbf{k} U(\mathbf{k}) \mathbf{k} e^{i \mathbf{k} \cdot \mathbf{r}(t)} \\
& \simeq-i \int d \mathbf{k} U(\mathbf{k}) \mathbf{k}\left\{1+i\left[\mathbf{k} \cdot \mathbf{r}_{1}(t)\right]\right\} e^{i \mathbf{k} \cdot \mathbf{r}_{0}(t)}
\end{aligned}
$$

The first and the second terms in the last part of Eq. (9) correspond to the first- $\left(\mathbf{f}_{1}\right)$ and the second-order $\left(\mathbf{f}_{2}\right)$ binary forces, respectively. We consider only the second-order binary force $\mathbf{f}_{2}$ and the corresponding force $\mathbf{F}_{2}$ with respect to the binary interaction since the averaged first-order force $\mathbf{F}_{1}$ (related to $\mathbf{f}_{1}$ ) vanishes due to symmetry reasons [21-25]. Within the secondorder perturbative treatment the cooling force can be represented as

$$
\begin{aligned}
\mathbf{F}_{2}= & Z k^{2} n_{e} \int d \mathbf{v}_{e} f\left(\mathbf{v}_{e}\right) v_{r} \int d^{2} \mathbf{s} \int d \mathbf{k} U(\mathbf{k}) \mathbf{k} \\
& \times \int_{-\infty}^{\infty}\left[\mathbf{k} \cdot \mathbf{r}_{1}(t)\right] e^{i \mathbf{k} \cdot \mathbf{r}_{0}(t)} d t .
\end{aligned}
$$

From Eq. (10) it is seen that the second-order cooling force, $\mathbf{F}_{2}$, is proportional to $Z^{2}$.

Substituting Eqs. (6) and (7) into Eq. (10) and writing the binary force in expression (7) in terms of Fourier transformed potential results in

$$
\begin{aligned}
\mathbf{F}_{2}= & \frac{i Z^{2} e^{4} n_{e}}{m} \int d \mathbf{v}_{e} f\left(\mathbf{v}_{e}\right) v_{r} \int d^{2} \mathbf{s} \int d \mathbf{k} d \mathbf{k}^{\prime} U(\mathbf{k}) U\left(\mathbf{k}^{\prime}\right) \mathbf{k} \\
& \times \int_{-\infty}^{\infty} e^{i \mathbf{k} \cdot \mathbf{r}_{0}(t)} d t \int_{-\infty}^{t} e^{i \mathbf{k}^{\prime} \cdot \mathbf{r}_{0}(\tau)} d \tau\left\{g_{0}(t-\tau)\right. \\
& \left.+\frac{g_{1}}{\omega_{c}} \sin \left[\omega_{c}(t-\tau)\right]-\frac{g_{2}}{\omega_{c}}\left\{1-\cos \left[\omega_{c}(t-\tau)\right]\right\}\right\},
\end{aligned}
$$

where $g_{0}=(\mathbf{k} \cdot \mathbf{b})\left(\mathbf{k}^{\prime} \cdot \mathbf{b}\right), g_{1}=\left(\mathbf{k} \cdot \mathbf{k}^{\prime}\right)-(\mathbf{k} \cdot \mathbf{b})\left(\mathbf{k}^{\prime} \cdot \mathbf{b}\right)$, $g_{2}=\left(\mathbf{k} \cdot\left[\mathbf{k}^{\prime} \times \mathbf{b}\right]\right)$. The time integral in Eq. (11) can be performed using the Fourier series expansion of the exponential function $e^{i z \sin (\omega t)}=\sum_{n=-\infty}^{\infty} J_{n}(z) e^{i n \omega t}$, where $J_{n}$ are the Bessel functions of the $n$th order (see, e.g., Ref. [38]). This yields

$$
\begin{aligned}
\mathbf{F}_{2}= & \frac{2 \pi i Z^{2} e^{4} n_{e}}{m} \int d \mathbf{v}_{e} f\left(\mathbf{v}_{e}\right) v_{r} \int d^{2} \mathbf{s} \int d \mathbf{k} d \mathbf{k}^{\prime} U(\mathbf{k}) U\left(\mathbf{k}^{\prime}\right) \mathbf{k} e^{i\left(\mathbf{k}+\mathbf{k}^{\prime}\right) \cdot \mathbf{R}_{0}} \sum_{n, m=-\infty}^{\infty} e^{i(n+m) \varphi} e^{-i n \theta-i m \theta^{\prime}} J_{n}\left(k_{\perp} a\right) J_{m}\left(k_{\perp}^{\prime} a\right) \delta\left[\zeta_{n}(\mathbf{k})\right. \\
& \left.+\zeta_{m}\left(\mathbf{k}^{\prime}\right)\right]\left\{-\frac{g_{0}}{\left[\zeta_{m}\left(\mathbf{k}^{\prime}\right)-i 0\right]^{2}}+\frac{g_{1}}{2 \omega_{c}}\left[\frac{1}{\zeta_{m+1}\left(\mathbf{k}^{\prime}\right)-i 0}-\frac{1}{\zeta_{m-1}\left(\mathbf{k}^{\prime}\right)-i 0}\right]\right. \\
& \left.+\frac{i g_{2}}{2 \omega_{c}}\left[\frac{2}{\zeta_{m}\left(\mathbf{k}^{\prime}\right)-i 0}-\frac{1}{\zeta_{m+1}\left(\mathbf{k}^{\prime}\right)-i 0}-\frac{1}{\zeta_{m-1}\left(\mathbf{k}^{\prime}\right)-i 0}\right]\right\}
\end{aligned}
$$

Here $\tan \theta=k_{y} / k_{x}, k_{\|}=(\mathbf{k} \cdot \mathbf{b})$, and $k_{\perp}$ are the components of $\mathbf{k}$ parallel and transverse to $\mathbf{b}$, respectively, $\zeta_{n}(\mathbf{k})=n \omega_{c}+\mathbf{k} \cdot \mathbf{v}_{r}$, and $\varphi$ is the initial phase of the electron as defined in the previous section. Note that expression (12) involves all cyclotron harmonics.

Next, we integrate with respect to the initial phase $\varphi$ and impact parameter $\mathbf{s}$. For that purpose we recall that the volume element $d \mathbf{v}_{e}$ can be represented in cylindrical coordinates as $d \mathbf{v}_{e}=d v_{e \|} v_{e \perp} d v_{e \perp} d \varphi$, where $v_{e \|}$ and $v_{e \perp}$ are the electron velocity components parallel and transverse to $\mathbf{b}$, respectively. The $\mathbf{s}$ integration is enabled by using the relation $e^{i \mathbf{k} \cdot \mathbf{R}_{0}}=e^{i \kappa_{\|} R_{0 \|}} e^{i \mathbf{k}_{\perp} \cdot \mathbf{s}}$, where $\kappa_{\|}=$ $\left(\mathbf{k} \cdot \mathbf{n}_{r}\right), \mathbf{\kappa}_{\perp}=\mathbf{k}-\mathbf{n}_{r}\left(\mathbf{k} \cdot \mathbf{n}_{r}\right)$, i.e. the component of $\mathbf{k}$ parallel and transverse to $\mathbf{n}_{r}$. Performing now the $\varphi$ and $\mathbf{s}$ integrations results in

$$
\begin{aligned}
\mathbf{F}_{2}= & -\frac{(2 \pi)^{5} Z^{2} e^{4} n_{e}}{2 m} \int_{-\infty}^{\infty} d v_{e \|} \int_{0}^{\infty} f\left(v_{e \|}, v_{e \perp}\right) v_{e \perp} d v_{e \perp} \\
& \times \int d \mathbf{k}|U(\mathbf{k})|^{2} \mathbf{k} \sum_{n=-\infty}^{\infty} J_{n}^{2}\left(k_{\perp} a\right)\left\{k_{\|}^{2} \delta^{\prime}\left[\zeta_{n}(\mathbf{k})\right]\right. \\
& \left.+\frac{k_{\perp}^{2}}{2 \omega_{c}}\left\{\delta\left[\zeta_{n+1}(\mathbf{k})\right]-\delta\left[\zeta_{n-1}(\mathbf{k})\right]\right\}\right\},
\end{aligned}
$$

where the prime indicates the derivative with respect to the argument. For deriving Eq. (13) we assumed an axially symmetric velocity distribution $f\left(\mathbf{v}_{e}\right)=f\left(v_{e \|}, v_{e \perp}\right)$ and used $\delta\left(\kappa_{\|}\right) \delta\left(\boldsymbol{\kappa}_{\perp}\right)=\delta(\mathbf{k})$.

The $n$ summation in Eq. (13) can be done using the summation formula for the Bessel functions [38]. We then obtain 


$$
\begin{aligned}
\mathbf{F}_{2}= & \frac{(2 \pi)^{4} Z^{2} e^{4} n_{e}}{m} \int_{-\infty}^{\infty} d v_{e \|} \int_{0}^{\infty} f\left(v_{e \|}, v_{e \perp}\right) v_{e \perp} d v_{e \perp} \\
& \times \int d \mathbf{k}|U(\mathbf{k})|^{2} \mathbf{k} \int_{0}^{\infty}\left[k_{\|}^{2}+k_{\perp}^{2} \frac{\sin \left(\omega_{c} t\right)}{\omega_{c} t}\right] \\
& \times J_{0}\left(2 k_{\perp} a \sin \frac{\omega_{c} t}{2}\right) \sin \left(\mathbf{k} \cdot \mathbf{v}_{r} t\right) t d t .
\end{aligned}
$$

This is a general expression for the magnetized cooling force acting on an individual ion. It has been derived within second-order perturbation theory but without any restriction on the strength of the magnetic field $B$. The limiting cases of Eq. (14) at vanishing $B$ and in the presence of an infinitely strong magnetic field are briefly studied in Sec. III A (see also Appendix A).

\section{Cooling force for a regularized and screened Coulomb potential}

In electron cooling of ion beams the velocity distribution of the electrons is anisotropic which is a typical situation for electron coolers. It is usually modeled by a twotemperature-anisotropic Maxwell distribution with different temperatures for the longitudinal and transverse degrees of freedom. The velocity distribution relevant for the averaging in Eq. (14) is thus given by

$$
f\left(v_{e \|}, v_{e \perp}\right)=\frac{1}{(2 \pi)^{3 / 2} v_{\mathrm{th} \perp}^{2} v_{\mathrm{th} \|}} e^{-v_{e \perp}^{2} / 2 v_{\mathrm{th} \perp}^{2}} e^{-v_{e \|}^{2} / 2 v_{\mathrm{th} \|}^{2}}
$$

where the thermal velocities are related to electron temperatures by $v_{\text {th } \perp}^{2}=T_{\perp} / m, v_{\text {th\|l }}^{2}=T_{\|} / m$ (here the temperatures are measured in energy units). In this case the transverse $\left(\mathbf{F}_{\perp}=\mathbf{F}-\mathbf{b} F_{\|}\right)$and longitudinal $\left(F_{\|}=\mathbf{b} \cdot \mathbf{F}\right)$ components of the cooling force (14) with Eq. (15) (we dropped the index 2 in $\mathbf{F}_{2}$ for simplicity) after velocity integrations (see Ref. [38]) can be represented in the forms

$\left\{\begin{array}{l}F_{\perp}\left(\mathbf{v}_{i}\right) \\ F_{\|}\left(\mathbf{v}_{i}\right)\end{array}\right\}=-\frac{8 Z^{2} e^{4} n_{e}}{m \omega_{c}^{2}} \frac{(2 \pi)^{4}}{4} \int_{0}^{\infty} d k_{\|} \int_{0}^{\infty} U^{2}(k) k_{\perp} d k_{\perp} \int_{0}^{\infty} e^{-\left(t^{2} / 2\right) k_{\|}^{2} a_{\|}^{2}} e^{-k_{\perp}^{2} a_{\perp}^{2}(1-\cos t)}\left(k_{\|}^{2}+k_{\perp}^{2} \frac{\sin t}{t}\right)\left\{\begin{array}{c}k_{\perp} \cos \left(k_{\|} a_{i \|} t\right) J_{1}\left(k_{\perp} a_{i \perp} t\right) \\ k_{\|} \sin \left(k_{\|} a_{i \|} t\right) J_{0}\left(k_{\perp} a_{i \perp} t\right)\end{array}\right\} t d t$

with $\mathbf{F}_{\perp}\left(\mathbf{v}_{i}\right)=\frac{\mathbf{v}_{i \perp}}{v_{i \perp}} F_{\perp}\left(\mathbf{v}_{i}\right)$. Here we have assumed a spherically symmetric potential $U(\mathbf{k})=U(k)$ and have introduced the thermal cyclotron radii of the electrons $a_{\perp}=v_{\text {th } \perp} / \omega_{c}, a_{\|}=v_{\text {th } \|} / \omega_{c}$, and $a_{i \perp}=v_{i \perp} / \omega_{c}, a_{i \|}=$ $v_{i \|} / \omega_{c}$. In general the cooling force is thus anisotropic with respect to the ion velocity $\mathbf{v}_{i}$.

For the Coulomb interaction $U(k)=U_{\mathrm{C}}(k)$, the full twodimensional integration over the $\mathbf{s}$ space results in a logarithmic divergence of the $\mathbf{k}$ integration in Eqs. (13) and (14). To cure this, cutoff parameters $k_{\min }$ and $k_{\max }$ must be introduced, see, e.g., Refs. [21-23] for details. Instead of doing so, we here employ the regularized screened potential $U(\mathbf{r})=U_{\mathrm{R}}(r)$ introduced in Sec. II A with the Fourier transform,

$$
U_{\mathrm{R}}(k)=\frac{2}{(2 \pi)^{2}}\left(\frac{1}{k^{2}+\lambda^{-2}}-\frac{1}{k^{2}+d^{-2}}\right)
$$

where $d^{-1}=\lambda^{-1}+\lambda^{-1}$.

Substituting the interaction potential (17) into Eq. (16) and performing the $k_{\|}$integration we arrive, after lengthy but straightforward calculations, at

$$
\begin{aligned}
F_{\|}\left(\mathbf{v}_{i}\right)= & -\frac{4 \sqrt{\pi} Z^{2} e^{4} n_{e}}{m v_{\mathrm{th} \|}^{2}} v_{\|} \int_{0}^{\infty} \frac{d t}{t} \int_{0}^{1} d \zeta \Phi[\psi(t, \zeta)] \exp \left[-v_{\|}^{2} \zeta^{2}-\frac{v_{\perp}^{2} \zeta^{2}}{G(t, \zeta)}\right] \frac{\zeta^{2}\left(1-\zeta^{2}\right)}{G(t, \zeta)}\left\{3-2 v_{\|}^{2} \zeta^{2}\right. \\
& \left.+\frac{2}{G(t, \zeta)}\left[1-\frac{v_{\perp}^{2} \zeta^{2}}{G(t, \zeta)}\right] \frac{\sin (\alpha t)}{\alpha t}\right\}, \\
F_{\perp}\left(\mathbf{v}_{i}\right)= & -\frac{4 \sqrt{\pi} Z^{2} e^{4} n_{e}}{m v_{\mathrm{th} \|}^{2}} v_{\perp} \int_{0}^{\infty} \frac{d t}{t} \int_{0}^{1} d \zeta \Phi[\psi(t, \zeta)] \exp \left[-v_{\|}^{2} \zeta^{2}-\frac{v_{\perp}^{2} \zeta^{2}}{G(t, \zeta)}\right] \frac{\zeta^{2}\left(1-\zeta^{2}\right)}{G^{2}(t, \zeta)}\left\{1-2 v_{\|}^{2} \zeta^{2}\right. \\
& \left.+\frac{2}{G(t, \zeta)}\left[2-\frac{v_{\perp}^{2} \zeta^{2}}{G(t, \zeta)}\right] \frac{\sin (\alpha t)}{\alpha t}\right\},
\end{aligned}
$$

where we have introduced the dimensionless quantities $v_{\|}=v_{i \|} / \sqrt{2} v_{\text {th } \|}, v_{\perp}=v_{i \perp} / \sqrt{2} v_{\text {th\| }}, \alpha=\omega_{c} \lambda / v_{\text {th } \|}$, and $\tau=$ $T_{\perp} / T_{\|}$is the anisotropy parameter of the electron beam. Here $\psi(t, \zeta)=\left(t^{2} / 2\right)\left(1-\zeta^{2}\right) / \zeta^{2}, G(t, \zeta)=\tau \Theta(t) \zeta^{2}+1-\zeta^{2}$, $\Theta(t)=\left(\frac{2}{\alpha t} \sin \frac{\alpha t}{2}\right)^{2}$, and 


$$
\Phi(z)=e^{-z}+e^{-x^{2} z}-\frac{2}{x^{2}-1} \frac{1}{z}\left(e^{-z}-e^{-x^{2} z}\right),
$$

where $x=\lambda / d=1+\lambda / \lambda$. Equations (18) and (19) for the parallel and transversal components of the drag force, respectively, are the main results of this paper. In the next section we compare systematically these expressions as well as general Eq. (14) with previous approaches.

\section{COMPARISON WITH PREVIOUS APPROACHES}

Previous theoretical expressions for the cooling force which have been extensively discussed by electron cooling community (see, e.g., Refs. [5,9] for a review) basically concern the two limiting cases of vanishing and infinitely strong magnetic fields. We therefore consider our previously presented approach in some detail for these two cases, first for arbitrary interactions $U(\mathbf{k})$ and electron distributions $f\left(\mathbf{v}_{e}\right)$ as given by Eq. (14) and later for the specific situation of the regularized interaction (17) and the velocity distribution (15) as given by Eqs. (18) and (19).

\section{A. Cooling force Eq. (14) at vanishing and infinitely strong magnetic fields}

For $B \rightarrow 0$, i.e. at vanishing magnetic field, $\sin \left(\omega_{c} t\right) /\left(\omega_{c} t\right) \rightarrow 1$ and the argument of the Bessel function in Eq. (14) should be replaced by $k_{\perp} v_{e \perp} t$. Then, denoting the second-order force at vanishing magnetic field as $\mathbf{F}_{0}$ and using an integral representation of the Bessel function $J_{0}$, one obtains

$$
\begin{aligned}
\mathbf{F}_{0}\left(\mathbf{v}_{i}\right)= & -\frac{(2 \pi)^{3} Z^{2} e^{4} n_{e}}{m} \int f\left(\mathbf{v}_{e}\right) d \mathbf{v}_{e} \int d \mathbf{k}|U(\mathbf{k})|^{2} k^{2} \mathbf{k} \\
& \times \frac{\partial}{\partial \omega} \int_{0}^{\infty} J_{0}\left(\mathbf{k}_{\perp} \cdot \mathbf{v}_{e \perp} t\right) \cos (\omega t) d t \\
= & \frac{4 \pi Z^{2} k^{4} n_{e}}{m} \frac{\partial}{\partial \mathbf{v}_{i}} \int G_{0}\left(\overline{\mathbf{v}}_{r}\right) f\left(\mathbf{v}_{e}\right) d \mathbf{v}_{e}
\end{aligned}
$$

with

$$
G_{0}\left(\overline{\mathbf{v}}_{r}\right)=\frac{(2 \pi)^{3}}{4} \int|U(\mathbf{k})|^{2} \delta\left(\mathbf{k} \cdot \overline{\mathbf{v}}_{r}\right) k^{2} d \mathbf{k} .
$$

Here $\omega=\mathbf{k} \cdot \mathbf{v}_{r}, \overline{\mathbf{v}}_{r}=\mathbf{v}_{r}+\mathbf{v}_{e \perp}=\mathbf{v}_{e}-\mathbf{v}_{i}, \mathbf{v}_{e}$ and $\overline{\mathbf{v}}_{r}$ are the three-dimensional electron and the ion-electron relative velocities, respectively. The other quantities in Eqs. (21) and (22) have been introduced in Sec. II. In particular, assuming spherically symmetric potential with $U(\mathbf{k})=$ $U(k)$, from Eq. (22) it is straightforward to obtain $G_{0}\left(\overline{\mathbf{v}}_{r}\right)=$ $G_{0}\left(\bar{v}_{r}\right)=\left(1 / \bar{v}_{r}\right) \mathcal{U}$ and thus

$$
\mathbf{F}_{0}\left(\mathbf{v}_{i}\right)=\frac{4 \pi Z^{2} e^{4} n_{e}}{m} \mathcal{U} \int \frac{\mathbf{v}_{e}-\mathbf{v}_{i}}{\left|\mathbf{v}_{e}-\mathbf{v}_{i}\right|^{3}} f\left(\mathbf{v}_{e}\right) d \mathbf{v}_{e},
$$

where $\mathcal{U}$ is the generalized Coulomb logarithm,

$$
\mathcal{U}=\frac{(2 \pi)^{4}}{4} \int_{0}^{\infty} U^{2}(k) k^{3} d k
$$

Employing the regularized and screened potential $U(k)$ given by Eq. (17), the generalized Coulomb logarithm is $\mathcal{U}=\mathcal{U}_{\mathrm{R}}=\Lambda(x)$ (see also Refs. [22-25]), where

$$
\Lambda(x)=\frac{x^{2}+1}{x^{2}-1} \ln x-1 .
$$

Taking the bare Coulomb interaction with $U(k)=$ $U_{\mathrm{C}}(k) \sim 1 / k^{2}$, Eq. (24) diverges logarithmically at $k \rightarrow 0$ and $k \rightarrow \infty$ and two cutoffs $k_{\min }=1 / r_{\max }$ and $k_{\max }=$ $1 / r_{\text {min }}$ must be introduced as discussed in Sec. II C. In this case the generalized Coulomb logarithm takes the standard form $\mathcal{U}=\mathcal{U}_{\mathrm{C}}=\ln \left(k_{\max } / k_{\min }\right)=\ln \left(r_{\max } / r_{\min }\right)$.

While the cooling force (23) is even at vanishing magnetic field anisotropic due to the anisotropic velocity distribution of the electrons, the asymptotic expression of (23) at high ion velocities is isotropic and can be easily derived by replacing $\overline{\boldsymbol{v}}_{r}=\left|\mathbf{v}_{e}-\mathbf{v}_{i}\right|$ with the ion velocity $\overline{\boldsymbol{v}}_{r} \simeq \boldsymbol{v}_{i}$ which results in

$$
\mathbf{F}_{0}\left(\mathbf{v}_{i}\right) \simeq-\frac{4 \pi Z^{2} k^{4} n_{e}}{m v_{i}^{2}} U \frac{\mathbf{v}_{i}}{v_{i}}
$$

At an infinitely strong magnetic field $B \rightarrow \infty$ the term in Eq. (14) proportional to $k_{\perp}^{2}$ and the argument of the Bessel function vanish since the cyclotron radius $a \rightarrow 0$. In this limit, denoting the force as $\mathbf{F}_{\infty}\left(\mathbf{v}_{i}\right)$, we arrive at

$$
\mathbf{F}_{\infty}\left(\mathbf{v}_{i}\right)=\frac{2 \pi Z^{2} e^{4} n_{e}}{m} \frac{\partial}{\partial \mathbf{v}_{i}} \int G_{\infty}\left(\mathbf{v}_{r}\right) f_{e}\left(\mathbf{v}_{e}\right) d \mathbf{v}_{e},
$$

where

$$
G_{\infty}\left(\mathbf{v}_{r}\right)=\frac{(2 \pi)^{3}}{2} \int|U(\mathbf{k})|^{2} \delta\left(\mathbf{k} \cdot \mathbf{v}_{r}\right) k_{\|}^{2} d \mathbf{k} .
$$

Again, assuming a spherically symmetric interaction potential from Eq. (28) we obtain $G_{\infty}\left(\mathbf{v}_{r}\right)=\left(v_{i \perp}^{2} / v_{r}^{3}\right) \mathcal{U}$, where $v_{i \perp}$ is the component of the ion velocity perpendicular to the magnetic field and $\mathcal{U}$ is given by Eq. (24). Inserting $G_{\infty}\left(\mathbf{v}_{r}\right)=\left(v_{i \perp}^{2} / v_{r}^{3}\right) \mathcal{U}$ into Eq. (27) then provides the two components of the cooling force,

$$
\begin{gathered}
F_{\infty \|}\left(\mathbf{v}_{i}\right)=\frac{6 \pi Z^{2} k^{4} n_{e}}{m} U \int \frac{v_{i \perp}^{2} v_{r \|}}{v_{r}^{5}} f_{e}\left(\mathbf{v}_{e}\right) d \mathbf{v}_{e}, \\
F_{\infty \perp}\left(\mathbf{v}_{i}\right)=\frac{2 \pi Z^{2} k^{4} n_{e}}{m} U \int \frac{v_{i \perp}\left(2 v_{r \|}^{2}-v_{i \perp}^{2}\right)}{v_{r}^{5}} f_{e}\left(\mathbf{v}_{e}\right) d \mathbf{v}_{e},
\end{gathered}
$$

where $v_{r \|}=v_{e \|}-v_{i \|}$. The corresponding high-velocity asymptotic expressions, replacing now $\mathbf{v}_{r}$ by $-\mathbf{v}_{i}$, are given by

$$
F_{\infty \|}\left(\mathbf{v}_{i}\right) \simeq-\frac{6 \pi Z^{2} e^{4} n_{e}}{m v_{i}^{2}} \mathcal{U} \frac{v_{i \perp}^{2} v_{i \|}}{v_{i}^{3}}
$$




$$
F_{\infty \perp}\left(\mathbf{v}_{i}\right) \simeq \frac{2 \pi Z^{2} k^{4} n_{e}}{m v_{i}^{2}} U \frac{v_{i \perp}\left(2 v_{i \|}^{2}-v_{i \perp}^{2}\right)}{v_{i}^{3}}
$$

Note that Eqs. (31) and (32) can be also obtained from Eqs. (29) and (30), respectively, in the case of a completely flattened distribution function of the electrons in the limit $T_{\|} \rightarrow 0$ when the distribution function (15) is given by a delta function with respect to $v_{e \|}$.

Equations (23), (29), and (30) and their asymptotic expressions for high velocities [Eqs. (26), (31), and (32), respectively] assuming the Coulomb interaction potential with $U=U_{\mathrm{C}}$ yield the cooling forces obtained previously in the cases of vanishing and infinitely strong magnetic fields, see e.g. [9], respectively. Equations (21) and (27) with a regularized interaction potential thus agree with the similar results derived by Derbenev and Skrinsky in Ref. [9] except for the different Coulomb logarithms $\mathcal{U}$. A more detailed discussion and comparison of $\mathcal{U}_{\mathrm{R}}=\Lambda(x)$ given by Eq. (25) and the standard Coulomb logarithm $\mathcal{U}_{\mathrm{C}}=\ln \left(r_{\max } / r_{\min }\right)$ can be found in Appendix B. We like to emphasize here that the Coulomb logarithm $\mathcal{U}_{\mathrm{R}}$ for the regularized interaction potential has the advantage to allow closed analytic expressions and converging integrals and avoids any introduction of lower and upper cutoffs "by hand" in order to restrict the domains of integration. Moreover, employing the bare Coulomb interaction may, as pointed out by Parkhomchuk [33], result in asymptotic expressions which are essentially different from Eqs. (29)-(32). In Appendix A we show how this is related to the divergent nature of the bare Coulomb interaction.

\section{B. Some limiting cases of Eqs. (18) and (19)}

More specifically we next discuss some asymptotic regimes of the cooling forces [Eqs. (18) and (19)] when assuming the regularized interaction (17) and the twotemperature velocity distribution (15). In the high-velocity limit where $v_{i}>\left(\omega_{c} \lambda, v_{\mathrm{th} \| ; \perp}\right)$ only small $t$ contribute to the cooling forces (18) and (19) due to the short time response of the electrons to the moving fast ion. In this limit we have $\sin (\alpha t) / \alpha t \rightarrow 1$ and $G(t, \zeta) \rightarrow \tau \zeta^{2}+1-\zeta^{2}$. The remaining $t$ integration can be performed explicitly. This integral is given by

$$
\begin{aligned}
\int_{0}^{\infty} \frac{d t}{t} \Phi[\psi(t, \zeta)] & =\lim _{\varepsilon \rightarrow 0^{+}} \frac{1}{2} \int_{\varepsilon}^{\infty} \frac{d z}{z} \Phi(z) \\
& =\lim _{\varepsilon \rightarrow 0^{+}} \frac{x^{2}+1}{2\left(\varkappa^{2}-1\right)}\left[E_{1}(\varepsilon)-E_{1}\left(\varkappa^{2} \varepsilon\right)\right]-1 \\
& \equiv \Lambda(x) .
\end{aligned}
$$

Here $z=\left(t^{2} / 2\right)\left(1 / \zeta^{2}-1\right)$ was introduced as a new variable of integration, the function $\Phi(z)$ is determined by Eq. (20), $E_{1}(z)=-\operatorname{Ei}(-z)$ is the exponential integral which behaves at small argument $(z \rightarrow 0)$ as $E_{1}(z) \simeq$ $\ln (1 / z)-\gamma$ [38], where $\gamma$ is the Euler's constant and $\Lambda(x)$ is the generalized Coulomb logarithm [Eq. (25)].
The remaining expressions do not depend on the magnetic field, i.e. $\omega_{c}$, as a natural consequence of the short time response of the magnetized electrons. In fact, $\sin (\alpha t) / \alpha t \rightarrow 1$ and $G(t, \zeta) \rightarrow \tau \zeta^{2}+1-\zeta^{2}$ and the related $t$ integration (33) are also valid for vanishing magnetic field $\alpha \rightarrow 0$. Changing now in the remaining $\zeta$ integrations the variable $\zeta \rightarrow \zeta /\left[\zeta^{2}+\tau\left(1-\zeta^{2}\right)\right]^{1 / 2}$ turns Eqs. (18) and (19), after some integration by parts, into

$$
\begin{aligned}
F_{\| ; \perp}\left(\mathbf{v}_{i}\right)= & -\frac{8 \sqrt{\pi} Z^{2} e^{4} n_{e}}{m v_{\mathrm{th} \| ; \perp}^{2}} \Lambda(x) v_{\| ; \perp} \\
& \times \int_{0}^{1} \exp \left[-\frac{v_{\|}^{2} \zeta^{2}}{\zeta^{2}+\tau\left(1-\zeta^{2}\right)}-\frac{v_{\perp}^{2} \zeta^{2}}{\tau}\right] \\
& \times \frac{\zeta^{2} d \zeta}{\left[\zeta^{2}+\tau\left(1-\zeta^{2}\right)\right]^{q}},
\end{aligned}
$$

where $q=3 / 2$ and $q=1 / 2$ for $F_{\|}\left(\mathbf{v}_{i}\right)$ and $F_{\perp}\left(\mathbf{v}_{i}\right)$, respectively. Here again the scaled ion velocities $v_{\|}=$ $v_{i \|} / \sqrt{2} v_{\text {th } \|}$ and $v_{\perp}=v_{i \perp} / \sqrt{2} v_{\text {th } \|}$ have been used. The cooling forces (34) are anisotropic with respect to the ion velocity $\mathbf{v}_{i}$ due to the anisotropic velocity distribution (15) of the electrons, and they represent the two limiting cases of Eqs. (18) and (19), namely high velocities at arbitrary magnetic field and arbitrary velocities at vanishing field. Of course, expression (34) can be also obtained by performing the remaining integration in the nonmagnetized cooling force (23) using the anisotropic velocity distribution (15) and $\mathcal{U}=\Lambda(x)$.

The cooling forces in (34) are additionally simplified when the transverse thermal velocity spread of the electrons $v_{\text {th } \perp}$ is much larger than the longitudinal one $v_{\text {th }} \|$ (i.e. $T_{\perp} \gg T_{\|}$or $\tau \gg 1$ ) which is a typical situation for electron coolers. In this case we have

$$
\begin{aligned}
F_{\| ; \perp}\left(\mathbf{v}_{i}\right)= & -\frac{8 \sqrt{\pi} Z^{2} k^{4} n_{e}}{m v_{\mathrm{th} \| ; \perp}^{2}} \Lambda(x) v_{\| ; \perp} \\
& \times \int_{0}^{1} \exp \left[-\frac{v_{\perp}^{2} \zeta^{2}}{\tau}-\frac{v_{\|}^{2} \zeta^{2}}{\tau\left(1-\zeta^{2}\right)}\right] \frac{\zeta^{2} d \zeta}{\left[\tau\left(1-\zeta^{2}\right)\right]^{q}},
\end{aligned}
$$

where the numerical factor $q$ is the same as introduced above.

A further increase of the ion velocity at $T_{\perp}>T_{\|}$finally yields

$$
\begin{aligned}
\mathbf{F}\left(\mathbf{v}_{i}\right) & \simeq-\frac{4 \pi Z^{2} e^{4} n_{e}}{m v_{i}^{2}} \Lambda(\varkappa) \frac{\mathbf{v}_{i}}{v_{i}}\left[\operatorname{erf}(v / \sqrt{\tau})-\frac{2}{\sqrt{\pi \tau}} v e^{-v^{2} / \tau}\right] \\
& \simeq-\frac{4 \pi Z^{2} e^{4} n_{e}}{m v_{i}^{2}} \Lambda(x) \frac{\mathbf{v}_{i}}{v_{i}},
\end{aligned}
$$

where $v^{2}=v_{\|}^{2}+v_{\perp}^{2}=v_{i}^{2} / 2 v_{\text {th\| }}^{2}$ and $\operatorname{erf}(z)$ is the error function. At sufficiently high velocities the cooling force (36) becomes isotropic and does not depend explicitly on the electron beam temperatures $T_{\|}$and $T_{\perp}$ [see the last part 
of Eq. (36)]. However, these temperatures can be involved in the generalized Coulomb logarithm in Eq. (36). Note that Eqs. (34)-(36) can be also derived from the general cooling force (21) inserting here the distribution function (15) and assuming the regularized interaction potential, i.e. $\mathcal{U}=\Lambda(x)$. Besides, Eq. (36) completely agrees with the asymptotic expression (26) by taking $\mathcal{U}=\Lambda(x)$.

At $B \rightarrow 0$ and small velocities $\left(v_{i}<v_{\text {th } \| ; \perp}\right)$ the cooling forces (34) become highly anisotropic and are given by

$\mathbf{F}\left(\mathbf{v}_{i}\right) \simeq-\frac{8 \sqrt{\pi} Z^{2} k^{4} n_{e}}{3 m v_{\mathrm{th} \|}^{2}} \Lambda(\varkappa)\left[\mathbf{n} v_{\|} \mathcal{B}_{1}(\tau)+\mathbf{v}_{\perp} \mathcal{B}_{2}(\tau)\right]$

where $\mathbf{n}$ is a unit vector along the axis of the electron beam anisotropy, and

$$
\begin{gathered}
\mathcal{B}_{1}(\tau)=\frac{3}{\tau-1}\left[1-\frac{1}{\sqrt{|1-\tau|}} p\left(\frac{1}{\sqrt{\tau}}\right)\right], \\
\mathcal{B}_{2}(\tau)=\frac{3}{2(\tau-1)}\left[\frac{\tau}{\sqrt{|1-\tau|}} p\left(\frac{1}{\sqrt{\tau}}\right)-1\right]
\end{gathered}
$$

with $\mathcal{B}_{1}(1)=\mathcal{B}_{2}(1)=1$, and

$$
p(x)= \begin{cases}\arccos x & x<1 \\ \ln \left(x+\sqrt{x^{2}-1}\right), & x>1\end{cases}
$$

Now we consider the situation when the magnetic field is very strong and the electron cyclotron radius is the smallest length scale, $\omega_{c} \lambda \gg\left(v_{i}, v_{\text {th } \| ; \perp}\right)$ and the friction force is only weakly sensitive to the transverse electron velocities and, hence, is affected only by their longitudinal velocity spread. In this limit $\sin (\alpha t) / \alpha t \rightarrow 0$ and $G(t, \zeta) \rightarrow$ $1-\zeta^{2}$ we obtain from Eqs. (18) and (19) after some lengthy but straightforward calculations,

$$
\begin{aligned}
F_{\| ; \perp}\left(\mathbf{v}_{i}\right)= & -\frac{4 \sqrt{\pi} Z^{2} k^{4} n_{e}}{m v_{\mathrm{th} \|}^{2}} \Lambda(x) v_{\| ; \perp} \int_{0}^{1} \exp \left(-v_{\|}^{2} \zeta^{2}\right. \\
& \left.-\frac{v_{\perp}^{2} \zeta^{2}}{1-\zeta^{2}}\right)\left(C-2 v_{\|}^{2} \zeta^{2}\right) \frac{\zeta^{2} d \zeta}{\left(1-\zeta^{2}\right)^{q}},
\end{aligned}
$$

where $C=3, C=1$ and $q=0, q=1$ for $F_{\|}\left(\mathbf{v}_{i}\right)$ and $F_{\perp}\left(\mathbf{v}_{i}\right)$, respectively. As expected the cooling forces in Eq. (41) are independent of the transverse temperature $T_{\perp}$ of the electrons except that $T_{\perp}$ may be involved in the Coulomb logarithm $\Lambda(x)$.

Expressions in Eq. (41) [as well as Eq. (34)] are very convenient for numerical calculations since they involve one-dimensional integrals with finite range. Similar expressions have been obtained by Pestrikov [39] where, however, the drag force involves an integral with infinite range. Up to the definition of the Coulomb logarithm [i.e., $\mathcal{U}=\Lambda(x)$ in our case and $\mathcal{U}=\mathcal{U}_{\mathrm{C}}$ in Ref. [39] ] both expressions are identical. This can be easily shown after changing the variable $\zeta$ in (41) to $x=\left[v_{\|}^{2} \zeta^{2}+\right.$ $\left.v_{\perp}^{2} \zeta^{2} /\left(1-\zeta^{2}\right)\right]^{1 / 2}$ and some subsequent rearrangement.
In particular, Eq. (41) is essentially simplified for a completely flattened distribution function of the electrons in the limit $T_{\|} \rightarrow 0$, i.e. a deltalike distribution function with respect to $v_{e \|}$ in Eq. (15). In this case it is straightforward to show that the parallel and transverse cooling forces in Eq. (41) are identical with Eqs. (31) and (32), respectively, with $\mathcal{U}=\Lambda(x)$.

In the high-velocity limit with $\omega_{c} \lambda \gg v_{i} \gg v_{\text {th\|; }}$, the parallel and transverse components of the cooling force, Eq. (41), become

$$
\begin{aligned}
F_{\|}\left(\mathbf{v}_{i}\right) \simeq & -\frac{\pi Z^{2} e^{4} n_{e}}{m v_{\mathrm{th} \|}^{2}} \Lambda(\varkappa) \frac{v_{\|}}{v^{3}}\left\{\frac{3 v_{\perp}^{2}}{v^{2}} \operatorname{erf}(v)\right. \\
& \left.+\frac{2}{\sqrt{\pi}} v e^{-v^{2}}\left[\frac{v_{\|}^{2}}{v^{2}}\left(3+2 v^{2}\right)-3\right]\right\}, \\
F_{\perp}\left(\mathbf{v}_{i}\right) \simeq & -\frac{\pi Z^{2} k^{4} n_{e}}{m v_{\mathrm{th} \|}^{2}} \Lambda(\chi) \frac{v_{\perp}}{v^{3}}\left\{\left(1-\frac{3 v_{\|}^{2}}{v^{2}}\right) \operatorname{erf}(v)\right. \\
+ & \left.\frac{2}{\sqrt{\pi}} v e^{-v^{2}}\left[\frac{v_{\|}^{2}}{v^{2}}\left(3+2 v^{2}\right)-1\right]\right\},
\end{aligned}
$$

With further increase of the ion velocity we can then neglect the exponential terms in Eqs. (42) and (43) while $\operatorname{erf}(v) \rightarrow 1$ which yields the asymptotic expressions Eqs. (31) and (32) [with $U=\Lambda(x)$ ], corresponding again as well to the often considered limit $T_{\|} \rightarrow 0$.

The forces given by Eqs. (42) and (43) [or Eqs. (31) and (32) with $\mathcal{U}=\Lambda(x)$ ] decay as the corresponding force (36) like $\sim v_{i}^{-2}$ with the ion velocity. But here, the parallel force (31) vanishes at $v_{i \perp}=0$ which is a consequence of the presence of a strong magnetic field, where the electrons move parallel to the magnetic field. If the ion moves also parallel to the field (i.e. $v_{i \perp}=0$ ) the averaged friction force must vanish within the $\mathrm{BC}$ treatment for symmetry reasons. The sign of the transverse force (32) depends on the angle between ion velocity and the magnetic field and tends to defocus ions with small transverse velocity, $v_{i \perp}<\sqrt{2} v_{i \|}$ while focusing them in the opposite case.

Finally, we also investigate the case of small velocities at strong magnetic fields. Introducing a new integration variable $y^{2}=v_{\perp}^{2} \zeta^{2} /\left(1-\zeta^{2}\right)$ in Eq. (41) and considering a small parallel velocity $\left(v_{\|} \ll 1\right)$ we arrive at

$$
\begin{aligned}
F_{\|}\left(\mathbf{v}_{i}\right) \simeq & \frac{4 \sqrt{\pi} Z^{2} k^{4} n_{e}}{m v_{\mathrm{th} \|}^{2}} \Lambda(x) v_{\|} \frac{\partial}{\partial \xi} \xi^{2} \frac{\partial^{2}}{\partial \xi^{2}}\left[e^{\xi} K_{0}(\xi)\right] \\
= & -\frac{2 \sqrt{\pi} Z^{2} k^{4} n_{e}}{m v_{\mathrm{th} \|}^{2}} \Lambda(x) v_{\|} v_{\perp}^{2} e^{v_{\perp}^{2} / 2}\left[\left(1+2 v_{\perp}^{2}\right) K_{1}\left(\frac{v_{\perp}^{2}}{2}\right)\right. \\
& \left.-\left(3+2 v_{\perp}^{2}\right) K_{0}\left(\frac{v_{\perp}^{2}}{2}\right)\right]
\end{aligned}
$$




$$
\begin{aligned}
F_{\perp}\left(\mathbf{v}_{i}\right) \simeq & -\frac{2 \sqrt{\pi} Z^{2} k^{4} n_{e}}{m v_{\mathrm{th} \|}^{2}} \Lambda(x) v_{\perp} \frac{\partial}{\partial \xi} \xi \frac{\partial}{\partial \xi}\left[e^{\xi} K_{0}(\xi)\right] \\
= & -\frac{2 \sqrt{\pi} Z^{2} e^{4} n_{e}}{m v_{\mathrm{th} \|}^{2}} \Lambda(x) v_{\perp} e^{v_{\perp}^{2} / 2} \\
& \times\left[\left(v_{\perp}^{2}+1\right) K_{0}\left(\frac{v_{\perp}^{2}}{2}\right)-v_{\perp}^{2} K_{1}\left(\frac{v_{\perp}^{2}}{2}\right)\right],
\end{aligned}
$$

where $\xi=v_{\perp}^{2} / 2$. As expected the parallel force is linear with respect to $v_{\|}$decreasing with an increasing transverse component $v_{\perp}$ of the ion velocity as $F_{\|} \sim v_{\perp}^{-3}$. The transverse force does not depend on $v_{\|}$in this limit and falls as $F_{\perp} \sim v_{\perp}^{-2}$ with the transverse velocity.

Considering now a small transverse velocity $v_{\perp} \ll 1$ yields

$$
\begin{gathered}
F_{\|}\left(\mathbf{v}_{i}\right)=-\frac{4 \sqrt{\pi} Z^{2} e^{4} n_{e}}{m v_{\mathrm{th} \|}^{2}} \Lambda(x) v_{\|} e^{-v_{\|}^{2}}, \\
F_{\perp}\left(\mathbf{v}_{i}\right)=-\frac{4 \sqrt{\pi} Z^{2} e^{4} n_{e}}{m v_{\mathrm{th} \|}^{2}} \Lambda(x) v_{\perp} \\
\times\left[e^{-v^{2}}\left(1-2 v^{2}\right) \ln \left(\frac{2 v}{v_{\perp}}\right)+H(v)\right],
\end{gathered}
$$

where two functions have been introduced:

$$
\begin{array}{r}
H(v)=\frac{1}{2} e^{-v^{2}}\left(2 v^{2}-1\right) \operatorname{Ei}\left(v^{2}\right)-1+Y^{\prime}(v), \\
Y(v)=-2 v^{3} \int_{0}^{1} e^{-v^{2} x^{2}} \ln (1-x) x d x
\end{array}
$$

Here $\operatorname{Ei}(z)$ is the exponential integral and the prime in Eq. (48) indicates the derivative with respect to the argument. The function $H(v)$ at small $(v \ll 1)$ and large $(v \gg 1)$ values of the argument behaves as $H(v) \simeq$ $\ln (1 / v)-1-\gamma / 2$ and $H(v) \simeq-\sqrt{\pi} / 2 v^{3}$, respectively, where $\gamma$ is Euler's constant. Now it is seen that at $v_{\perp} \ll 1$ the parallel force (46) decays exponentially (i.e. much faster) with $v_{\|}$in contrast to the power law decays considered above. The transverse force (47), on the other hand, leads at low transverse ion velocities $v_{\perp}$ to a term which behaves as $\sim v_{\perp} \ln \left(1 / v_{\perp}\right)$. Thus the friction coefficient in the transverse direction diverges logarithmically at small $v_{\perp}$. This is a quite unexpected behavior compared to the well-known linear velocity dependence without magnetic field (see asymptotic expressions above). Finally, with increasing parallel velocity $v_{\|}$of the ion the logarithmic term vanishes exponentially and the transverse force behaves as $F_{\perp} \sim v_{\perp} / v^{3}$.

\section{FEATURES OF THE COOLING FORCES EQS. (18) AND (19) AND COMPARISON WITH CTMC SIMULATIONS}

In this section we study some general properties of the cooling forces on individual ions resulting from the BC approach by evaluating Eqs. (18) and (19) numerically.
We consider both the effects of the magnetic field and of a variation of the shape of the electron distribution on the cooling forces at various transverse velocities $v_{i \perp}$ of the ions. The density $n_{e} \simeq 10^{6} \mathrm{~cm}^{-3}$ and the temperatures $T_{\|} \simeq 0.1 \mathrm{meV}$ and $T_{\perp} \simeq 0.11 \mathrm{eV}$ of the electron beam are the same as in the experiments at the ESR storage ring [28-30] (see also Sec. V for further details) and are typical for many other electron cooling experiments. Thus, the electron beam is strongly anisotropic with $T_{\perp} \gg T_{\|}$. As an example we choose $\mathrm{C}^{6+}$ and $\mathrm{Xe}^{54+}$ fully stripped ions for our calculations. In all examples considered below the regularization parameter $\lambda_{0}=10^{-9} \mathrm{~m}$ and thereby meets the condition $\lambda_{0} \ll b_{0}(0)$, i.e. $\lambda_{0}$ does not affect noticeably the cooling forces (18) and (19) at low and medium velocities as shown in Appendix C.

For a $\mathrm{BC}$ description beyond the perturbative regime a fully numerical treatment is required. In the present cases of interest such a numerical evaluation of the cooling forces is rather intricate, but can be successfully implemented by classical trajectory Monte Carlo (CTMC) simulations [14-16]. In the CTMC method the trajectories for the ionelectron relative motion are calculated by a numerical integration of the equations of motion (1). The cooling force is then deduced by averaging over a large number (typically $10^{5}-10^{6}$ ) of trajectories employing a Monte Carlo sampling for the related initial conditions. For a more detailed description of the method we refer to Refs. [23-25]. Both the analytic perturbative treatment and the nonperturbative numerical CTMC simulations are based on the same BC picture and use the same effective spherical screened interaction $U(r)$. The following comparison of both these approaches thus essentially intends to check the validity and range of applicability of the perturbative approach as it has been outlined in the preceding sections.

First we consider the effect of the strength of the magnetic field on the second-order cooling forces. In Figs. 1-3 the parallel ( $-F_{\|}$, left panels) and transverse $\left(-F_{\perp}\right.$, right panels) cooling forces (in $\mathrm{eV} / \mathrm{m}$ ) given by Eqs. (18) and (19), respectively, are plotted vs ion beam parallel velocity $v_{i \|}$ (in $\mathrm{m} / \mathrm{s}$ ) for $\mathrm{C}^{6+}$ ions and at fixed $v_{i \perp}=0.1 v_{\text {th }}$ (Fig. 1), $v_{i \perp}=v_{\text {th } \|}$ (Fig. 2), and $v_{i \perp}=10 v_{\text {th } \|}$ (Fig. 3) and for various values of the magnetic field and are shown as the lines without symbols. The two limiting cases of vanishing $(B=0)$ and infinitely strong $(B=\infty)$ magnetic fields are obtained from Eqs. (34) and (41), respectively. Note that the transverse velocity of the ion is rather small, i.e. $v_{i \perp} \ll v_{\text {th } \perp}$, in these examples (Figs. 1-3), which results in a very small transverse cooling force at $B=0$. Indeed comparing Eqs. (34) and (41), one concludes that typically $F_{\infty \perp} / F_{0 \perp} \sim T_{\perp} / T_{\|} \gg 1$ at small and intermediate velocity range, where $F_{\infty \perp}$ and $F_{0 \perp}$ are the transverse cooling forces at $B=\infty$ and $B=0$, respectively. Therefore in the right panels of Figs. $1-3$ the values of the transverse forces at $B=0$ are increased by some appropriate (large) factors. The filled symbols in Figs. 1-3 

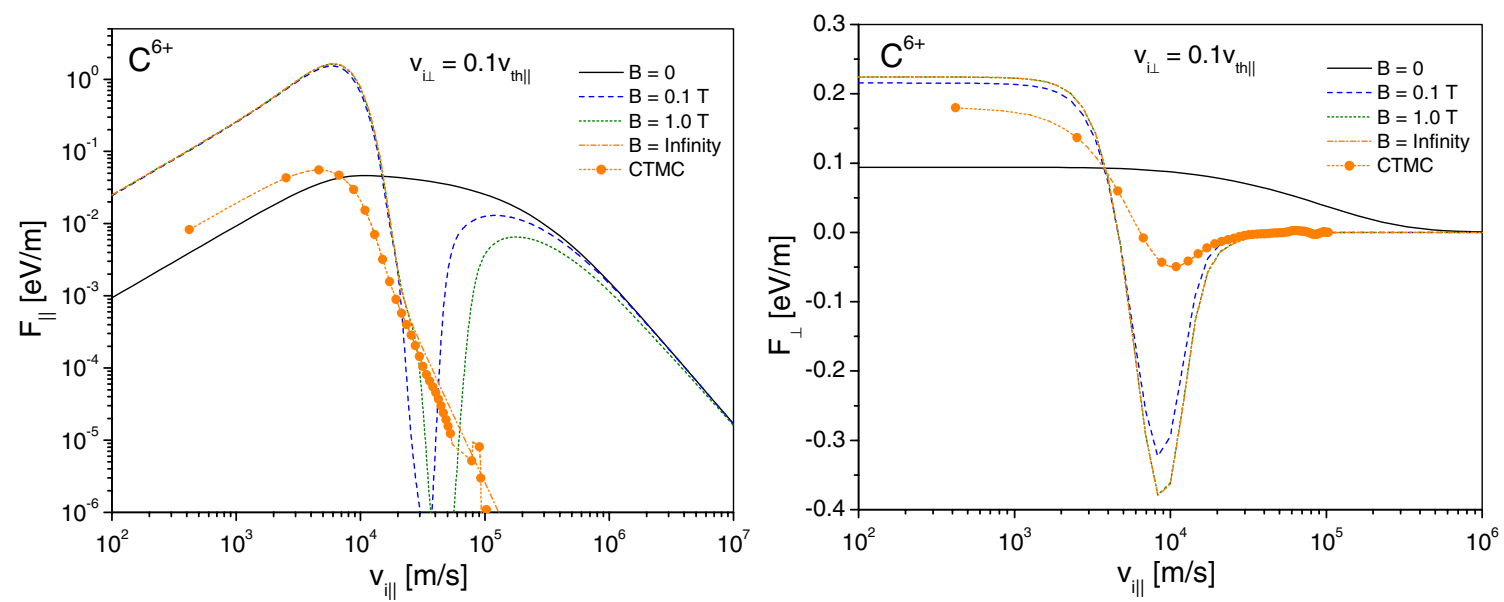

FIG. 1. Longitudinal ( $-F_{\|}$, left panel) and transverse $\left(-F_{\perp}\right.$, right panel) cooling forces (with minus signs and in $\left.\mathrm{eV} / \mathrm{m}\right) \mathrm{for} \mathrm{C}^{6+}$ fully stripped ions as a function of the ion velocity $v_{i \|}$ (in $\mathrm{m} / \mathrm{s}$ ) and for fixed $v_{i \perp}=0.1 v_{\text {th } \|}$ and $\lambda=\lambda_{\mathrm{D} \|}$. The theoretical cooling forces (18) and (19) are calculated for $\lambda_{0}=10^{-9} \mathrm{~m}$ (see Appendix B for details) and for an electron beam with $n_{e}=10^{6} \mathrm{~cm}^{-3}$, $T_{\perp}=0.11 \mathrm{eV}$, and $T_{\|}=0.1 \mathrm{meV}$ in a magnetic field of $B=0$ (solid line), $0.1 \mathrm{~T}$ (dashed line), $1 \mathrm{~T}$ (dotted line), and $B=\infty$ (dash-dotted line). The CTMC results for the $B=\infty$ case are shown by the filled circles. Note that in the right panel the transverse force for $B=0$ is increased by a factor $10^{3}$.
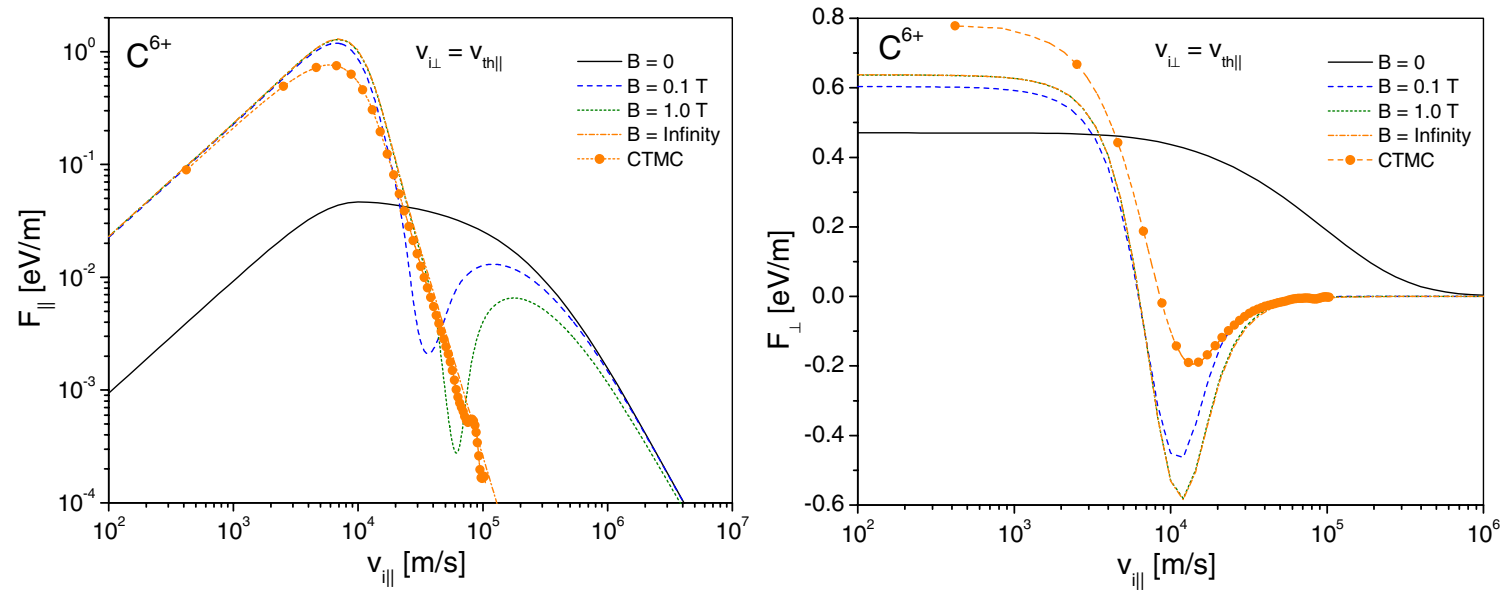

FIG. 2. Same as in Fig. 1 but for fixed $v_{i \perp}=v_{\text {th\| } \|}$. Note that in the right panel the transverse force for $B=0$ is increased by a factor $5 \times 10^{2}$.
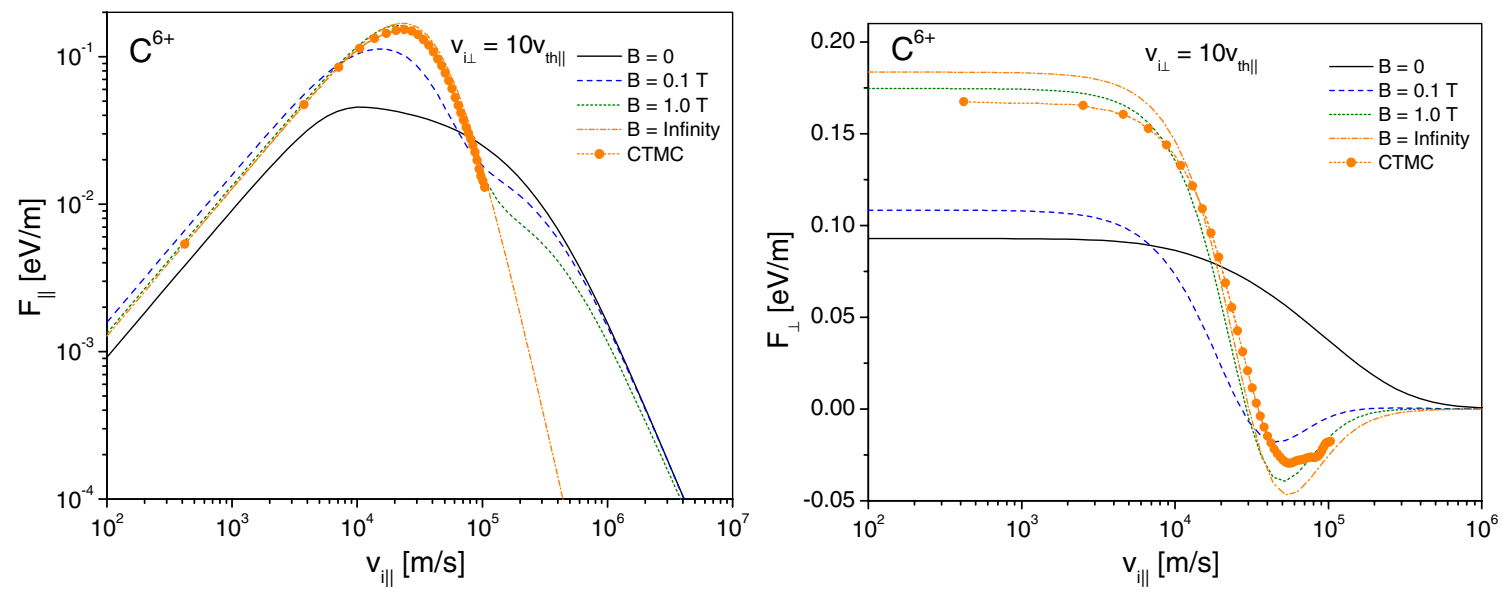

FIG. 3. Same as in Fig. 1 but for fixed $v_{i \perp}=10 v_{\text {th } \|}$. Note that in the right panel the transverse force for $B=0$ is increased by a factor 10 . 
represent the results of the CTMC simulations obtained for an infinitely strong magnetic field $(B=\infty)$; CTMC results for a finite magnetic field are shown later in Figs. 5-7. For simplifying the comparison, in both treatments, the perturbative $\mathrm{BC}$ and the CTMC calculations, the screening length was fixed by $\lambda=\lambda_{\mathrm{D} \|}$, independently of the strength of the magnetic field, where $\lambda_{\mathrm{D} \|}=v_{\mathrm{th} \|} / \omega_{p}$ is the longitudinal Debye length and $\omega_{p}$ is the electron plasma frequency. For the perturbative cooling forces we also employed the velocity-dependent regularization parameter $\lambda\left(v_{i \|}\right)$ of the interaction potential as discussed in Appendix B.

Compared to the unmagnetized case with $B=0$ (solid curves in Figs. 1-3) the magnetic field increases the cooling force $F_{\|}$at low velocities while reducing it at high velocities. Furthermore, the deviations of the parallel cooling force from the unmagnetized regime are stronger at smaller $v_{i \perp}$, that is, the cooling force is less sensitive to $B$ at large $v_{i \perp}$ in all shown cases.

A somewhat different picture is observed for the absolute value of the transverse force, $\left|F_{\perp}\right|$ (Figs. 1-3, right panels), when turning on the magnetic field from $B=0$ to $B=\infty$. The force $F_{\perp}$ is much more sensitive to the variation of $B$ (compared to the parallel force $F_{\|}$) and $\left|F_{\perp}\right|$ is strongly increased by the magnetic field in the whole parallel velocity range and for any transverse velocity $v_{i \perp}$. While $F_{\|}$is almost independent of the transverse ion velocity at small $v_{i \perp} \ll v_{\text {th } \|}$ the transverse force $F_{\perp}$ first shows a linear increase with $v_{i \perp}$ [see Eq. (19) and the right panels of Figs. 1 and 2] but is reduced again by a further increase of $v_{i \perp}$ (Fig. 3, right panel). In addition, for both $F_{\|}$and $F_{\perp}$, a rather weak magnetic field may produce significant deviations from the $B=0$ regime at small and intermediate velocities $v_{i \|}$ and $v_{i \perp}$. At high velocities and a strong magnetic field $(B=1 \mathrm{~T})$ the cooling force $F_{\|}$ strongly deviates from the extreme case with $B=\infty$, which is, however, not accessible for the present experiments at storage rings. At arbitrarily strong but finite magnetic field and sufficiently high velocities, $v_{i} \gg$ $\left(\omega_{c} \lambda, v_{\text {th } \| ; \perp}\right)$, the cooling force (18) converges to the parallel unmagnetized force, Eq. (34), which is the leading order term $\mathcal{O}\left(v_{i}^{-2}\right)$ of the high-velocity expansion of Eq. (18), while, as discussed in Sec. III B, the regime of infinitely strong magnetic field, Eq. (41), is reached for lower velocities $v_{i} \ll \omega_{c} \lambda$. At high velocities and strong magnetic field, the cooling force given by Eq. (18) thus deviates systematically from the regime of infinitely strong magnetic field, Eq. (41).

Another interesting feature of the parallel cooling force (18) observed in Figs. 1-3, in particular at small transverse velocities $v_{i \perp}$, is the formation of two maxima at parallel $\left(v_{i \|} \sim v_{\text {th } \|}\right)$ and transverse $\left(v_{i \|} \sim v_{\text {th } \perp}\right)$ electron thermal velocities with the formation of a corresponding (deep) minimum. Here, the maximum at $v_{i \|} \sim v_{\text {th }}$ is systematically larger than the second one at higher velocities $v_{i \|} \sim v_{\text {th } \perp}$. While the position of the low-velocity maximum of the force $F_{\|}$is almost independent on the strength of the magnetic field, the high-velocity maximum is reduced and its position is shifted towards higher $v_{i \|}$ at increasing $B$ making the force minimum deeper. A further increase of the magnetic field $(B>1 \mathrm{~T})$ and finally the transition to the regime $B=\infty$ results in a less structured shape of the parallel force. Increasing, however, the transverse ion velocity $v_{i \perp}$ reduces the depth of the force minimum and results at $v_{i \perp} \gg v_{\text {th\| }}$ in a smoother shape with only one maximum, see Fig. 3 (left panel).

Figures 1-3 also clearly demonstrate focusing or "antifriction" (given by the negative values shown on the right panels of Figs. $1-3$ by positive values) and the change of the sign of the transverse force $F_{\perp}$ which become more pronounced with increasing magnetic field. Similar features for the transfers force have been reported in Refs. [40,41] using VORPAL simulations. The asymptotic expression (32) [or the more accurate asymptotic Eq. (43)] predicts that the change of the sign of the force $F_{\perp}$ occurs at $v_{i \|}=v_{i \perp} / \sqrt{2}$ which corresponds to a constant (i.e. independent of $B$ and $v_{i \perp}$ ) angle $\vartheta=\arctan \sqrt{2}$ between the magnetic field $\mathbf{B}$ and the ion velocity $\mathbf{v}_{i}$. Let us recall, however, that the asymptotic expression (32) derived in the case of an infinitely strong magnetic field is valid either at vanishing longitudinal velocity spread $\left(T_{\|} \rightarrow 0\right)$ of the electrons or at high velocities $v_{i \|} \gg v_{\text {th } \|}$ of the ion [see Eq. (43)]. Our numerical calculations of the second-order forces $F_{\perp}$ shown in Figs. 1-3 (right panels) also shows an almost constant angle $\vartheta$, i.e. independent of $B$, which now, however, depends on the transverse velocity $v_{i \perp}$. At smaller $v_{i \perp}$ the angle $\vartheta$ when the force $F_{\perp}$ changes the sign is much smaller than the value predicted by the asymptotic Eq. (32) (Fig. 1, right panel) but with increasing $v_{i \perp}$ it converges to the constant value given above (Fig. 3, right panel).

Comparisons of the cooling forces determined by the CTMC simulations and the second-order perturbative treatment [Eq. (41)] at infinitely strong magnetic field are presented in Figs. 1-3 by the filled symbols and the dashdotted lines, respectively. It is seen that in general the perturbative treatment overestimates the CTMC results for both components of the cooling force which is, however, more pronounced for $F_{\perp}$. On the other hand, it is clearly observed that in the regimes of large parallel velocity $v_{i \|}$ and for arbitrary $v_{i \perp}$ the second-order perturbative treatment agrees almost perfectly (within the unavoidable numerical fluctuations) with the CTMC results. Increasing, however, the transverse velocity $v_{i \perp}$ of the ion, one arrives at the regime where the conditions of the applicability of the perturbative treatment (see, e.g., the brief discussion in Sec. II A) are less critical and an excellent agreement between second-order BC and CTMC is observed in the whole parallel velocity range as shown, for instance, in the left panels of Figs. 2 and 3. Obviously the agreement between both approaches is, in general, better for 
the parallel forces. In addition, similar to the second-order $\mathrm{BC}$ approach the CTMC also demonstrates the formation of antifriction for the transverse force $F_{\perp}$. That is, the secondorder BC qualitatively captures the velocity domain where the force changes the sign although it does not predict correctly the magnitude of the force at small $v_{i \perp}$.

Next we also look for some complementary information about the cooling forces (18) and (19), and plot in Fig. 4 these forces on $\mathrm{C}^{6+}$ ions vs ion beam parallel velocity at fixed $v_{i \perp}=v_{\text {th\| }}$ (cf. Figs. 2 and 4) but for a different shape of the distribution function of the electrons with smaller $T_{\perp}=10^{-2} \mathrm{eV}$. That is, Fig. 4 is equivalent to Fig. 2 except of the smaller transverse thermal velocity $v_{\text {th } \perp \text { and }}$ cyclotron radius $a_{\perp}=v_{\text {th } \perp} / \omega_{c}$ of the electrons in Fig. 4. This change of the transverse temperature has little influence on both components of the magnetized cooling force, only the minimum of the parallel force is increased by decreasing $T_{\perp}$. At this smaller cyclotron radius $a_{\perp}$ of the electrons the transverse force is almost independent of $B$ and converges to the regime of infinitely strong magnetic field as shown in Fig. 4 (right panel). On the other hand, both components of the unmagnetized force (solid lines) are strongly increased at smaller temperature $T_{\perp}$.

The regimes of an infinitely strong magnetic field where we already compared the CTMC simulations with the second-order perturbative treatment are, however, far from being accessible by any realistic scenario at storage rings. Thus, we also present results for the second-order parallel $\left(-F_{\|}\right)$cooling forces (in $\mathrm{eV} / \mathrm{m}$, lines without symbols) given by Eq. (18) as functions of the ion parallel
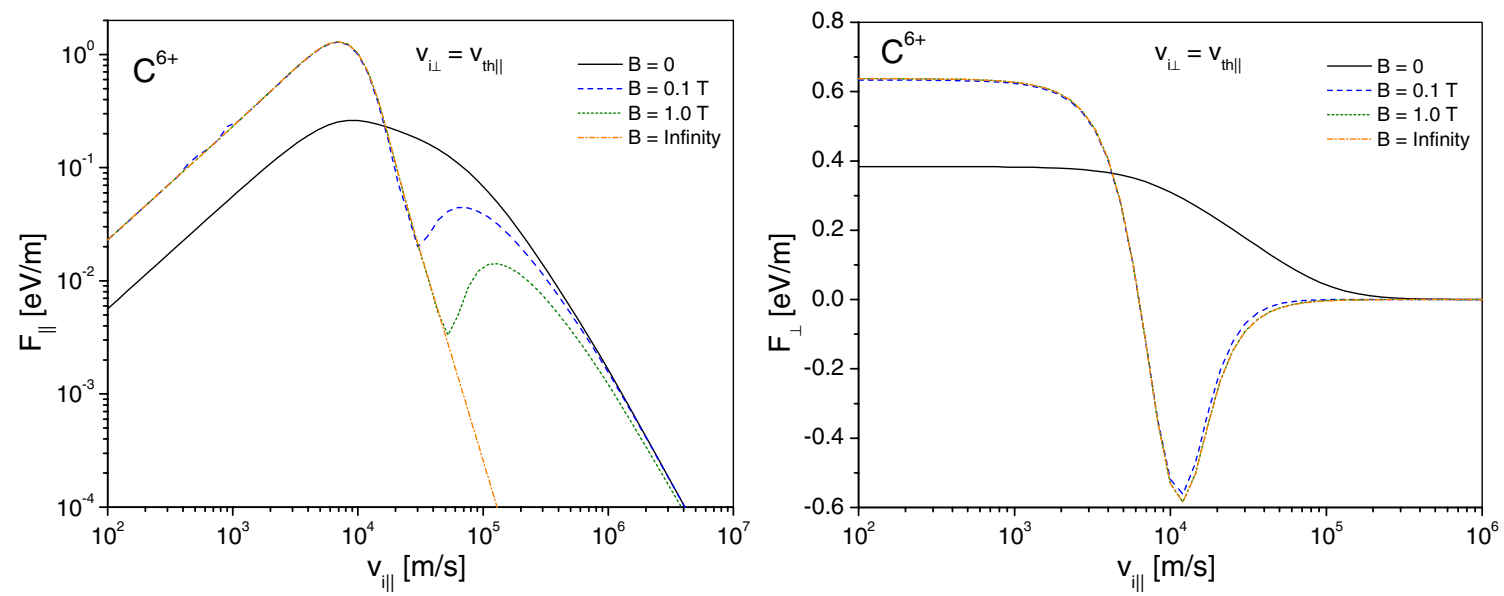

FIG. 4. Same as in Fig. 2 with $v_{i \perp}=v_{\text {th\| }}$ but for $T_{\perp}=10^{-2} \mathrm{eV}\left(T_{\perp}=10^{2} T_{\|}\right)$. Note that in the right panel the transverse force for $B=0$ is increased by a factor 20 .
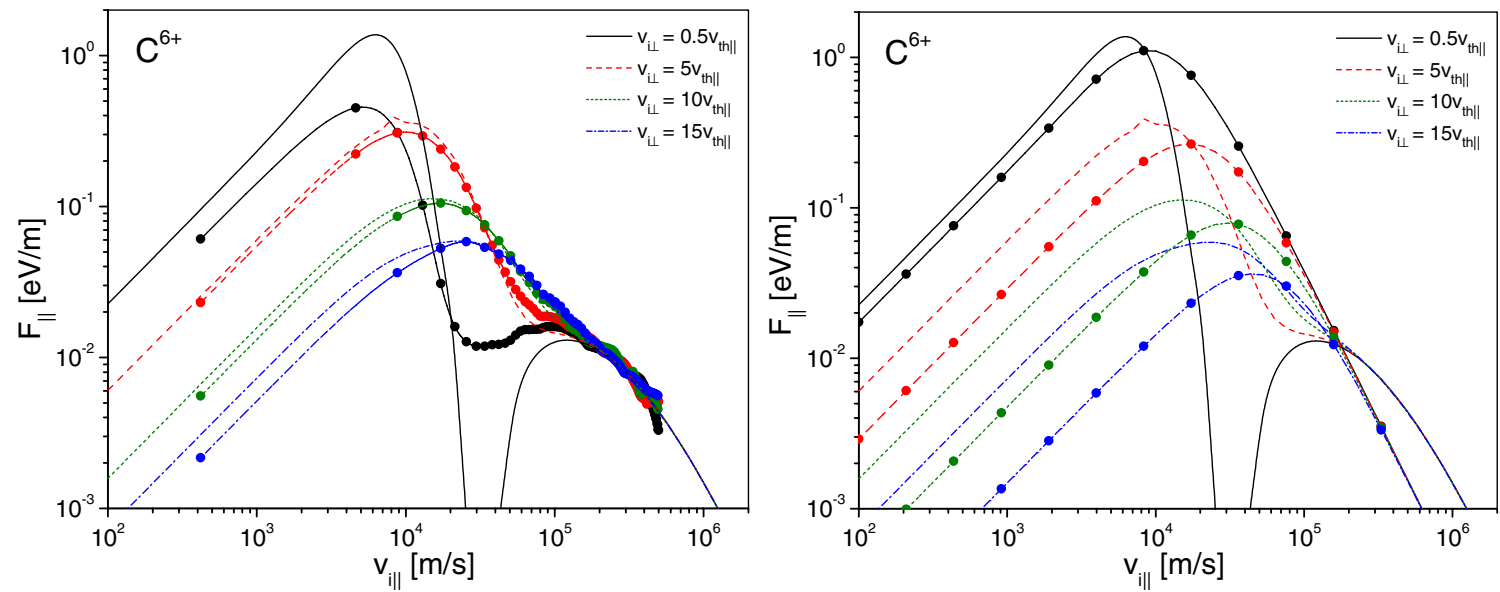

FIG. 5. Left panel: Longitudinal cooling force $-F_{\|}$(in $\mathrm{eV} / \mathrm{m}$ ) for the $\mathrm{C}^{6+}$ ion as function of $v_{i \|}$ (in $\mathrm{m} / \mathrm{s}$ ) for $\lambda=\lambda_{\mathrm{D} \|}$ and $v_{i \perp}=$

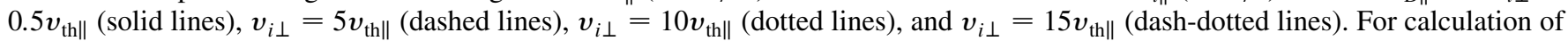
the theoretical cooling force (18) (the lines without symbols), the same set of parameters are used as in Fig. 1 with the value of a magnetic field $B=0.1 \mathrm{~T}$. The CTMC results are shown by the lines with filled circles. Right panel: Same as in the left panel but the present perturbative treatment (represented in the left panel by the lines without symbols) is compared with the PF (the lines with symbols) as given by Eq. (50) for $v_{\text {eff }}=2 v_{\text {th } \|}$. See the text for further details. 

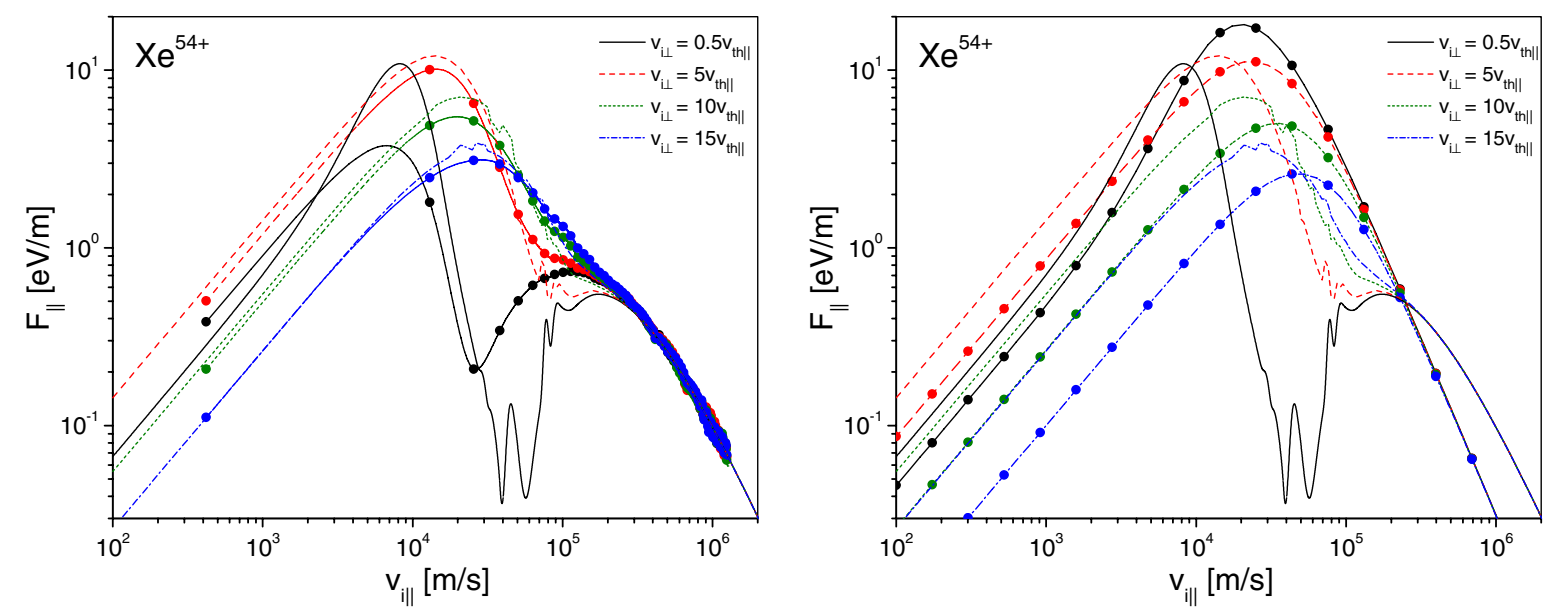

FIG. 6. Same as in Fig. 5 but for $\mathrm{Xe}^{54+}$ ion. In the right panel the PF is evaluated with $v_{\text {eff }}=3.5 v_{\text {th } \|}$.
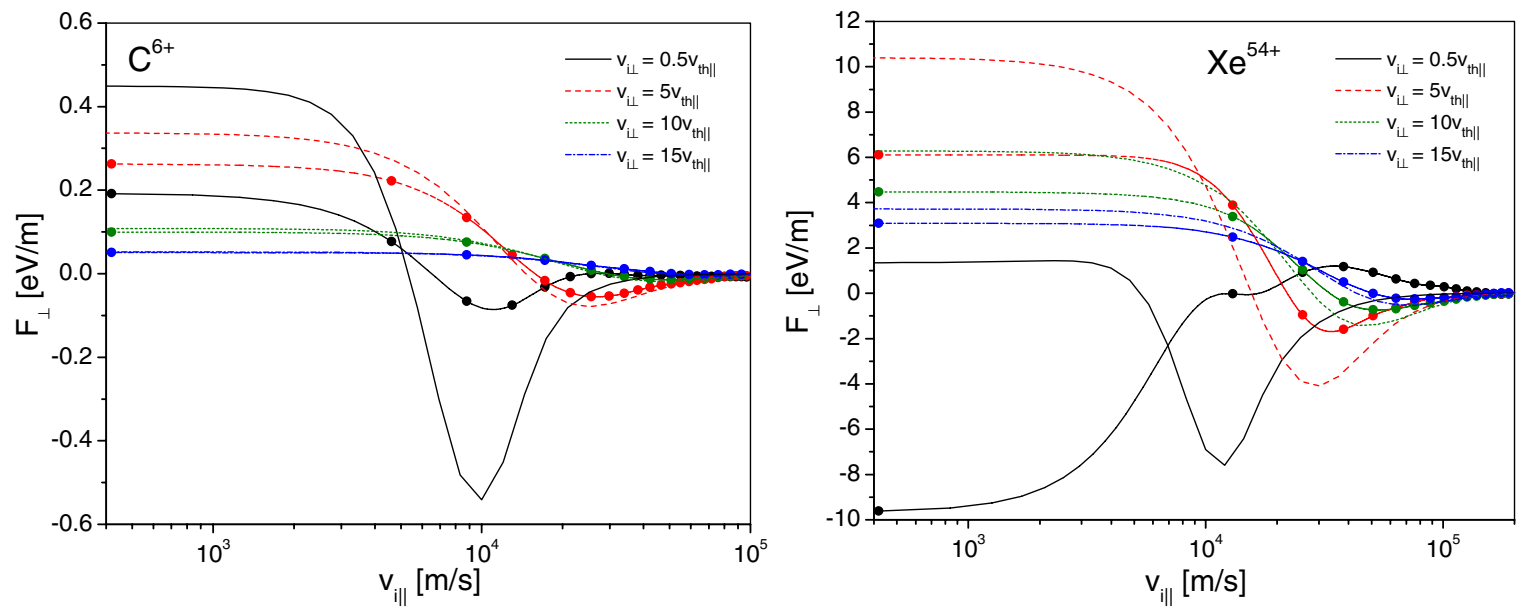

FIG. 7. Same as in the left panels of Figs. 5 and 6 for $\mathrm{C}^{6+}$ and $\mathrm{Xe}^{54+}$ ions, respectively, but for the transverse force $-F_{\perp}$.

velocity $v_{i \|}$ (in $\mathrm{m} / \mathrm{s}$ ) in Figs. 5 and 6 , now for the fully stripped ions $\mathrm{C}^{6+}$ and $\mathrm{Xe}^{54+}$ at a finite magnetic field $B=$ $0.1 \mathrm{~T}$ and fixed $v_{i \perp}=0.5 v_{\text {th } \|}$ (solid lines), $v_{i \perp}=5 v_{\text {th } \|}$ (dashed lines), $v_{i \perp}=10 v_{\text {th } \|}$ (dotted lines), and $v_{i \perp}=$ $15 v_{\text {th } \|}$ (dash-dotted lines). The density and the parallel and transverse temperatures of the electron beam are the same as in the experiments at the ESR storage ring [28-30] (see also Fig. 1). Again, the filled symbols in the left panels of Figs. 5 and 6 represent the results of the CTMC simulations obtained for a magnetic field $B=0.1 \mathrm{~T}$. As before the screening length is here fixed by the constant value $\lambda=$ $\lambda_{\mathrm{D} \|}$ and the velocity-dependent regularization parameter $\lambda\left(v_{i \|}\right)$ needed in the perturbative BC is again as determined in Appendix B.

We also compared our model to the empirical formula for the parallel cooling force on a single ion,

$F_{\|}\left(\mathbf{v}_{i}\right)=-\frac{4 \pi n_{e} Z^{2} k^{4}}{m} \frac{v_{i \|}}{\left(v_{i \|}^{2}+v_{i \perp}^{2}+v_{\mathrm{eff}}^{2}\right)^{3 / 2}} \ln \left(1+\frac{s_{\max }}{s_{\min }+a_{\perp}}\right)$, as proposed by Parkhomchuk [31,32] [for simplicity this formula is abbreviated below as PF (Parkhomchuk's formula)]. Here $s_{\min }=|Z| e^{2} / m\left(v_{i}^{2}+v_{s}^{2}\right)$ and $s_{\max }=$ $\left(v_{i}^{2}+v_{s}^{2}\right)^{1 / 2} / \omega_{p}$ are the dynamical minimal and maximal impact parameters, respectively, $a_{\perp}$ is the cyclotron radius of the electrons, and $v_{\text {eff }}$ is an effective electron velocity related to the transverse magnetic and electric fields in the electron cooler (see Refs. [31,32]) which can be viewed as a fitting parameter, and $v_{s}$ is a characteristic thermal velocity, as discussed in Appendix B. For consistency with our BC approach and the CTMC simulations, however, we evaluated Eq. (50) by fixing $s_{\max }$ also to the static screening length $s_{\max }=\lambda_{\mathrm{D} \|}$. In the right panels of Figs. 5 and 6 , the PF cooling forces $F_{\|}$(lines with symbols, taking the rather small values $v_{\text {eff }}=2 v_{\text {th } \|}$ and $v_{\text {eff }}=3.5 v_{\text {th } \| \text {, }}$ respectively) are compared to the perturbative treatment, which is represented by the same curves (lines without symbols) as in the left panels of Figs. 5 and 6.

Furthermore, in Fig. 7, we also compare second-order and CTMC results (lines without and with filled symbols, 
respectively) for the transverse cooling forces $F_{\perp}$ for $\mathrm{C}^{6+}$ (left panel) and $\mathrm{Xe}^{54+}$ (right panel) ions for the same set of parameters as in the left panels of Figs. 5 and 6.

Figures 5-7 demonstrate basically the same features for the second-order cooling forces as already discussed in connection with Figs. 1-4. Regarding the parallel components of these forces there is a quite good overall qualitative agreement with the CTMC results. In particular, the CTMC shows at small $v_{i \perp}$ the formation of two maxima of $F_{\|}$, a higher one at $v_{i \|} \sim v_{\text {th } \|}$ and a lower one at $v_{i \|} \sim v_{\text {th } \perp}$, as it is also predicted by the perturbative $\mathrm{BC}$. The perturbative $\mathrm{BC}$ overestimates, however, the cooling force at low velocities as well as the depth of its minimum in between of the two maxima, with the tendency that the quantitative agreement with the CTMC is generally strongly improved with increasing velocities $v_{i \|}$ and $v_{i \perp}$ (see the left panels of Figs. 5 and 6). This is basically what is to be expected for a perturbative treatment which should work best in the high-velocity weak coupling regime as defined by Eq. (5). Essentially the same behavior we also observed for the transverse force $F_{\perp}$, shown in Fig. 7, although the quantitative agreement with CTMC is less distinct here than for $F_{\|}$. But again, the perturbative $\mathrm{BC}$ qualitatively captures well the velocity domains where the transverse force is either negative or positive and the agreement between perturbative $\mathrm{BC}$ and CTMC is clearly improved with weaker electron-ion coupling, that is, for lower $Z$ and larger $v_{i \|}$ and $v_{i \perp}$. The only exception is here the case of the highly charged $\mathrm{Xe}^{54+}$ at the lowest $v_{i \perp}=0.5 v_{\text {th } \|}$ (Fig. 7, right panel) where the CTMC exhibits a completely different behavior of $F_{\perp}$. But this is also the case of the highest electron-ion coupling parameter where, according to Eq. (5), the applicability of a perturbative treatment becomes questionable. In addition, for heavy ions, like e.g. $\mathrm{Xe}^{54+}$, and low $v_{i \perp}$, that is, for the highest electron-ion coupling, and in the vicinity of the minimum of $F_{\|}$at intermediate $v_{i \|}$ the $\mathrm{BC}$ treatment starts to predict unphysical results like the sawtooth structure of $F_{\|}$emerging in this domain; see the solid lines in Fig. 6.

Finally, we turn to the comparisons of our model given by Eq. (18) and the PF [Eq. (50)] both shown in the right panels of Figs. 5 and 6. The considerable differences between Eq. (18) and the PF now clearly reveal the different nature of both these approaches. The empirical PF curve shows just some shift when varying the parameters, namely $v_{i \perp}$, while essentially retaining its shape. The perturbative $\mathrm{BC}$ model as well as the nonperturbative CTMC which are based on the full equations of motion in the presence of a magnetic field exhibit a much more intricate structure, in particular at small $v_{i \perp}$, the formation of two maxima of the parallel force $F_{\|}$at parallel and transverse electron thermal velocities. And the PF only covers the parallel force and does not offer any description of the transverse force.

\section{COOLING FORCE FOR A MAXWELLIAN ION DISTRIBUTION}

Up to now we considered the magnetized cooling force acting on the individual ion interacting with an electron beam with anisotropic velocity distribution. But often, the measured longitudinal cooling force represents an average over the drag forces on individual ions. Thus, the cooling force has to be interpreted as the average $\left\langle F_{\|}\left(\mathbf{v}_{i}\right)\right\rangle=\mathcal{F}$ of the component $F_{\|}\left(\mathbf{v}_{i}\right)$ of the drag force parallel to the beam axis (and the magnetic field) over the ion distribution $f_{i}\left(v_{i \|}, v_{i \perp}\right)$ in the beam (see, e.g., Refs. [40-43]), that is,

$\mathcal{F}=2 \pi \int_{-\infty}^{\infty} d v_{i \|} \int_{0}^{\infty} f_{i}\left(v_{i \|}, v_{i \perp}\right) F_{\|}\left(v_{i \|}, v_{i \perp}\right) v_{i \perp} d v_{i \perp}$.

\section{A. Averaged cooling force}

Modeling the ion beam by the anisotropic Maxwell distribution,

$f_{i}\left(v_{i \|}, v_{i \perp}\right)=\frac{1}{(2 \pi)^{3 / 2} \sigma_{\perp}^{2} \sigma_{\|}} e^{-v_{i \perp}^{2} / 2 \sigma_{\perp}^{2}} e^{-\left(v_{i \|}-\tilde{v}_{i \|}\right)^{2} / 2 \sigma_{\|}^{2}}$,

an analytic expression for the average $\mathcal{F}$, Eq. (51), over the BC drag force $F_{\|}\left(\mathbf{v}_{i}\right)$ given by (16) can be derived by substituting Eqs. (16) and (52) into Eq. (51) and then integrating over $v_{i \perp}$ and $v_{i \|}$, which yields

$$
\begin{aligned}
\mathcal{F}(u)= & -\frac{8 Z^{2} e^{4} n_{e} \lambda^{2}}{m v_{\mathrm{th} \|}^{2}} \frac{(2 \pi)^{4}}{4} \int_{0}^{\infty} k_{\|} d k_{\|} \int_{0}^{\infty} U^{2}(k) k_{\perp} d k_{\perp} \\
& \times \int_{0}^{\infty} e^{-\frac{t^{2}}{2} \lambda^{2}\left[k_{\|}^{2} \delta_{\|}^{2}+k_{\perp}^{2} D(t)\right]}\left(k_{\|}^{2}+k_{\perp}^{2} \frac{\sin (\alpha t)}{\alpha t}\right) \\
& \times \sin \left(\sqrt{2} k_{\|} \lambda u t\right) t d t .
\end{aligned}
$$

The introduced dimensionless parameters $D(t)=$ $\delta^{2}+\tau \Theta(t), u=\tilde{v}_{i \|} / \sqrt{2} v_{\text {th } \|}, \delta_{\|}^{2}=1+\sigma_{\|}^{2} / v_{\text {th } \|}^{2}$, and $\delta=$ $\sigma_{\perp} / v_{\text {th\| }}$ are related to the distribution of the ion beam (52), where $\sigma_{\perp}^{2}=(1 / 2)\left\langle v_{i \perp}^{2}\right\rangle=T_{i \perp} / M, \quad \sigma_{\|}^{2}=\left\langle v_{i \|}^{2}\right\rangle-\tilde{v}_{i \|}^{2}=$ $T_{i \|} / M$ with the effective transverse $\left(T_{i \perp}\right)$ and longitudinal $\left(T_{i \|}\right)$ temperatures of the ions and the ion mass $M$, and $\tilde{v}_{i \|}$ is the average $\mathrm{cm}$ velocity of the ion beam with respect to the electron beam.

Finally, substituting the interaction potential (17) into Eq. (53) and performing the $k_{\|}$integration we arrive at

$$
\begin{aligned}
-\mathcal{F}(u)= & \frac{4 \sqrt{\pi} Z^{2} k^{4} n_{e}}{m v_{\mathrm{th} \|}^{2}} u \int_{0}^{\infty} \frac{d t}{t} \int_{0}^{1} d \zeta \Phi[\psi(t, \zeta)] \\
& \times \exp \left(-\frac{u^{2} \zeta^{2}}{P^{2}(\zeta)}\right) \frac{1-\zeta^{2}}{P^{3}(\zeta) Q(t, \zeta)}\left[\frac{\zeta^{2}}{P^{2}(\zeta)}\left(3-\frac{2 u^{2} \zeta^{2}}{P^{2}(\zeta)}\right)\right. \\
& \left.+\frac{2 \zeta^{2}}{Q(t, \zeta)} \frac{\sin (\alpha t)}{\alpha t}\right]
\end{aligned}
$$

with $P(\zeta)=\left(\delta_{\|}^{2} \zeta^{2}+1-\zeta^{2}\right)^{1 / 2}, Q(t, \zeta)=D(t) \zeta^{2}+1-\zeta^{2}$. All other quantities have already been introduced in Sec. II C [see above Eq. (20)]. 
While Eq. (54) has to be evaluated numerically, a closed analytic expression can be derived for the limiting cases of (54) at high and low velocities and strong magnetic fields. In the high-velocity limit with $\tilde{v}_{i \|}>\left(\omega_{c} \lambda, v_{\text {th } \| ; \perp}, \sigma_{\| ; \perp}\right)$ only small $t$ contribute to the cooling force (54) and $\sin (\alpha t) / \alpha t \rightarrow 1$ and $Q(t, \zeta) \rightarrow \delta_{\perp}^{2} \zeta^{2}+1-\zeta^{2}$, where $\delta_{\perp}^{2}=D(0)=\delta^{2}+\tau$. At a sufficiently large ion beam velocity, Eq. (54) then turns into

$$
\begin{aligned}
-\mathcal{F}(u) & \simeq \frac{2 \pi Z^{2} e^{4} n_{e}}{m v_{\mathrm{th} \|}^{2}} \Lambda(x) \frac{1}{u^{2}}\left[\operatorname{erf}\left(\frac{u}{\delta_{\perp}}\right)-\frac{2}{\sqrt{\pi}} \frac{u}{\delta_{\perp}} e^{-u^{2} / \delta_{\perp}^{2}}\right] \\
& \simeq \frac{2 \pi Z^{2} k^{4} n_{e}}{m v_{\mathrm{th} \|}^{2}} \frac{\Lambda(x)}{u^{2}}
\end{aligned}
$$

where the force decreases as $\mathcal{F}(u) \sim u^{-2}$ with the beam velocity.

At very strong magnetic fields, when the electron cyclotron radius is the smallest length scale and $\sin (\alpha t) / \alpha t \rightarrow 0$, $Q(t, \zeta) \rightarrow \delta^{2} \zeta^{2}+1-\zeta^{2}$, and in the high-velocity limit with $\omega_{c} \lambda \gg \tilde{v}_{i \|} \gg\left(v_{\text {th } \| ; \perp}, \sigma_{\| ; \perp}\right)$, we obtain

$$
\begin{aligned}
-\mathcal{F}(u) \simeq & \frac{3 \pi Z^{2} e^{4} n_{e}}{m v_{\mathrm{th} \|}^{2}} \Lambda(x) \frac{\delta^{2}}{u^{4}} \\
& \times\left[\operatorname{erf}\left(\frac{u}{\delta}\right)-\frac{2}{3 \sqrt{\pi}} \frac{u}{\delta}\left(3+\frac{2 u^{2}}{\delta^{2}}\right) e^{-u^{2} / \delta^{2}}\right] \\
\simeq & \frac{3 \pi Z^{2} e^{4} n_{e}}{m v_{\mathrm{th} \|}^{2}} \Lambda(x) \frac{\delta^{2}}{u^{4}} .
\end{aligned}
$$

There is an important difference if we compare Eqs. (56) and (55). The force (56) decays as $\mathcal{F}(u) \sim u^{-4}$ much faster than in Eq. (55). The velocity of the beam in Eq. (56) is large but is restricted to the value $\omega_{c} \lambda$, i.e. $1 \ll u \ll \omega_{c} \lambda$. Thus it cannot be arbitrarily large. The velocity in Eq. (55) is arbitrarily large but now restricted below, $\tilde{v}_{i \|} \gg \omega_{c} \lambda$, i.e. the magnetic field there cannot be arbitrarily large.

Considering on the other hand also the case of small velocities $u \ll 1$ at strong magnetic fields, Eq. (54) becomes

$$
\begin{gathered}
-\mathcal{F}(u) \simeq \frac{8 \sqrt{\pi} Z^{2} e^{4} n_{e}}{5 m v_{\mathrm{th} \|}^{2} \delta_{\|} \delta^{2}} \Lambda(x) u \mathcal{P}\left(\frac{\delta_{\|}}{\delta}\right), \\
\mathcal{P}(x)=\frac{5}{2\left(1-x^{2}\right)^{2}}\left[x^{2}+2-\frac{3 x}{\sqrt{\left|1-x^{2}\right|}} p(x)\right],
\end{gathered}
$$

and $p(x)$ is given by Eq. (40). As expected the low-velocity cooling force [Eq. (57)] strongly depends on the details of the distribution functions of electrons and ions.

\section{B. Comparison with experiment}

With the theoretical formalism presented above, we now compare the cooling forces on the ions resulting from our analytical approach, Eq. (54), with available experimental data.
Measurements of the cooling forces have been performed at several storage rings, like e.g. at the ESR at GSI [28-30]. In these experiments a so-called cooling force is extracted, which can be viewed as a stopping force averaged over the ion distribution in the beam and the electron distribution. As an example we focus on the measurements of longitudinal cooling forces for different fully stripped heavy ions as conducted at the electron cooler of the ESR storage ring. Two different methods have been used here to determine the cooling force. At low ion velocities the cooling force is extracted from the equilibrium between cooling and longitudinal heating with rf noise. At high relative velocities between the rest frames of the beams, the cooling force is deduced from the momentum drift of the ion beam after a rapid change of the electron energy. Details of these methods as well as the experimental conditions and observations are given in Refs. [28-30]. The measured cooling forces are shown in Fig. 8 (filled circles) for various fully stripped ions.

The electron beam in these experiments has a density of $n_{e} \simeq 10^{6} \mathrm{~cm}^{-3}$ and can be described by an anisotropic velocity distribution (15) with $T_{\perp}=m v_{\mathrm{th} \perp}^{2} \simeq 0.11 \mathrm{eV}$ and $T_{\|}=m v_{\text {th\|l }}^{2} \simeq 0.1 \mathrm{meV}$ as inferred from corresponding measurements. The strength of the magnetic guiding field was $B=0.1 \mathrm{~T}$. The measured longitudinal cooling force represents an average over the stopping forces on individual ions. For a comparison with the theoretical model (54) the cooling force is thus interpreted as the average $\left\langle F_{\|}\right\rangle$of the component $F_{\|}$of the stopping force (14) parallel to the beam axis (and the magnetic field) over

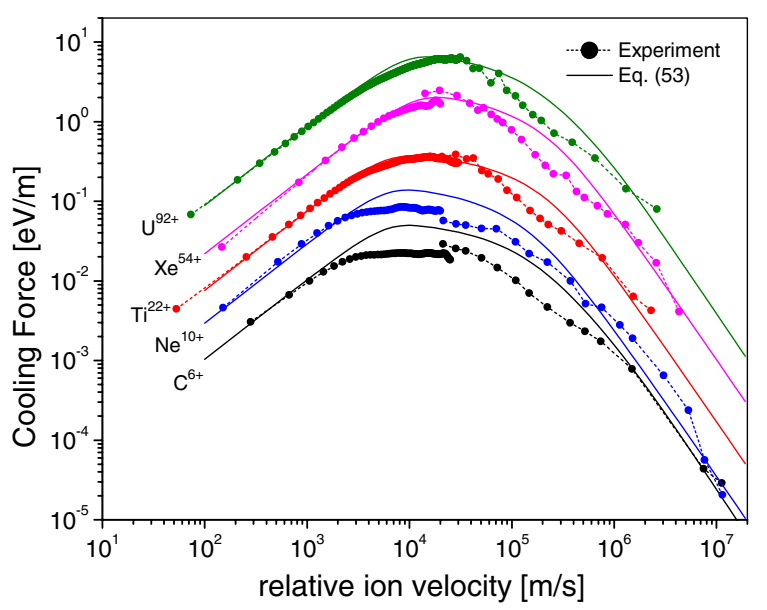

FIG. 8. Longitudinal cooling force (in $\mathrm{eV} / \mathrm{m}$ ) for various fully stripped ions as a function of the relative ion velocity (in $\mathrm{m} / \mathrm{s}$ ). Filled circles: Experimental data from measurements at the electron cooler of the ESR storage ring [28-30]. Solid curves: Eq. (54). The theoretical predictions of the cooling force are calculated for an electron beam with $n_{e}=10^{6} \mathrm{~cm}^{-3}, T_{\perp}=$ $0.11 \mathrm{eV}$, and $T_{\|}=0.1 \mathrm{meV}$ in a magnetic field of $B=0.1 \mathrm{~T}$, and are fitted to the experimental results at low relative velocities by treating the quantities $\sigma_{\|}, \sigma_{\perp}$ as free parameters (see the text for details). 
the ion distribution $f_{i}\left(v_{i \|}, v_{i \perp}\right)$ in the beam (see also Refs. [40-43]).

For low ion velocities this average is taken with respect to the transverse ion velocity only and the cooling force depends on the parallel ion velocity, i.e. $\left\langle F_{\|}\right\rangle=\left\langle F_{\|}\right\rangle\left(v_{i \|}\right)$. In the experimental procedure used for high ion velocities the cooling force is an average over the complete ion distribution. This average $\left\langle F_{\|}\right\rangle=\left\langle F_{\|}\right\rangle\left(\left\langle v_{i \|}\right\rangle\right)$ depends now on the velocity of the $\mathrm{cm}$ of the ion beam relative to the rest frame of the electron beam $\left\langle v_{i \|}\right\rangle$. Both velocities are denoted as relative ion velocity in Fig. 8. To perform the average the distribution $f_{i}\left(v_{i \|}, v_{i \perp}\right)$ must be known. However, in Refs. [28-30] this distribution was not determined in detail, but there exists an estimate of the beam angular divergence $\left\langle\theta_{i}\right\rangle \lesssim 0.5 \mathrm{mrad}$ [29]. This yields after transformation to the rest frame of the ion beam for the transverse ion velocities $\left\langle v_{i \perp}\right\rangle \simeq v_{i \perp}^{*} \equiv \beta \gamma c\left\langle\theta_{i}\right\rangle$, where $\beta$, $\gamma$ are the relativistic factors related to the beam velocity in the lab frame and $c$ is the speed of light. For the measurements at hand with an ion energy of $250 \mathrm{MeV} / \mathrm{u}$ $(\beta=0.615, \gamma=1.268)$ this results in $v_{i \perp}^{*} \leq 1.17 \times 10^{5} \mathrm{~m} / \mathrm{s}$.

Now we turn to the present expression for the cooling force (54) which is shown as solid curves in Fig. 8. The velocity spread, i.e. the widths $\sigma_{\perp}, \sigma_{\|}$of the ion distribution (52), was treated as a free parameter to fit the $\mathrm{BC}$ stopping force to the experimental data. As the cooling force $\mathcal{F}$ is rather sensitive to a variation of $\sigma_{\perp}$ at low parallel velocities $v_{i \|}$ this fit is done for the linear increase of the cooling force at low relative velocities. The velocity spread of the ion beam in transverse direction used in obtaining the solid curves is $3.5 v_{i \perp}^{*} \lesssim \sigma_{\perp} \lesssim 4.5 v_{i \perp}^{*}$ with $\left\langle\theta_{i}\right\rangle \simeq 0.2 \mathrm{mrad}\left(v_{i \perp}^{*} \simeq 4.7 \times 10^{4} \mathrm{~m} / \mathrm{s}\right)$ which is in $\operatorname{good}$ agreement with the estimated beam divergence $\left\langle\theta_{i}\right\rangle$. The spread in the longitudinal direction is here typically $\sigma_{\|} \lesssim$ $10^{-2} \sigma_{\perp}$ as it usually occurs in many experimental situations (see, e.g., [3,40-43] and references therein), in particular at the ESR storage ring [28-30]. In the examples considered here the regularization parameter $\lambda_{0}$ varies within $10^{-10}-10^{-7} \mathrm{~m}$ with $\lambda_{0} \ll b_{0}(0)$, i.e. $\lambda_{0}$ does not affect noticeably the cooling force (54) at low and medium velocities (see Appendix C). The BC model [Eq. (54)] well agrees with the experimental cooling force at low and high velocities but somewhat overestimates the cooling force at medium velocities. These deviations are more pronounced for lower ion charge states, but the overall behavior is essentially independent of the ion charge.

For the parameters and conditions of the considered experiments and taking into account the averages over the electron and ion distribution functions, the domain of hard collisions and relative velocities which violate the condition for a perturbative treatment $|Z| e^{2} / m v_{0}^{2} \lambda<1$ [see Eq. (5)] is rather small and thus ensures the overall applicability of our model in the present regimes. More specific, the related characteristic velocities $\left(|Z| e^{2} / m \lambda\right)^{1 / 2}$ are here $8.7 \times 10^{2} \mathrm{~m} / \mathrm{s}$ for $Z=6$ and $3.4 \times 10^{3} \mathrm{~m} / \mathrm{s}$ for
$Z=92$ (taking for low ion velocities the static screening length $\lambda=\bar{\lambda}_{\mathrm{D}}$ defined in Appendix C). This has to be contrasted with a typical lower limit of the relative ionelectron velocity $v_{0}$ which is given by the parallel thermal electron velocity $v_{\text {th } \|} \simeq 4.2 \times 10^{3} \mathrm{~m} / \mathrm{s}$ when assuming low ion velocities and neglecting the transverse component of $v_{0}$. The deviations of the perturbative $\mathrm{BC}$ cooling force (54) (solid curves) from the ESR data (filled circles) we therefore mainly ascribe to the rather unknown distribution function of the ions in the beam which has been modeled here in the form of an anisotropic Maxwell distribution (52). Indeed the actual velocity spread in ion beams may essentially differ from the Maxwellian (52) and, in particular, in some cases the recorded profiles are parabolic rather than Maxwellian [40-43] (see also Ref. [3]). For a comprehensive comparison with the measurements and a critical evaluation of theoretical approaches a detailed knowledge of the ion distribution is indispensable.

\section{SUMMARY}

In this paper we presented and discussed analytic expressions for calculating the cooling force on ions in a model of binary collisions (BC) between ions and magnetized electrons within second-order perturbative treatment. This has been done within the framework of an improved $\mathrm{BC}$ theory which involves all cyclotron harmonics of the electrons' helical motion and which is valid for any strength of the magnetic field and in regimes where a perturbative treatment is applicable. The cooling force is explicitly calculated for a regularized and screened Coulomb potential. Closed expressions have been derived first for monochromatic electron beams, which have been folded with the velocity distributions of the electrons and ions. The resulting cooling force is evaluated for anisotropic Maxwell velocity distributions of the electrons and ions. A number of limiting and asymptotic regimes of low and high velocities as well as vanishing and strong magnetic fields have been studied. The given results show that the present model of the cooling force is very sensitive to the velocity spreads of the electrons and ions at small relative velocities. Main limitations and uncertainties of the present BC model are: (1) the approximations concerning the electron and ion distribution functions, (2) the use of a spherically symmetric effective interaction accounting for screening effects and hard collisions, and (3) the underlying perturbative expansion of the equations of motion. The latter can be well justified as long as the majority of the electron-ion collisions which contribute to the averaged final cooling force clearly meets the condition of a weak perturbation; see Eq. (5). The use of an effective interaction, on the other hand, and the proper choice of a velocitydependent screening length clearly needs still some support from a comparison with full self-consistent simulation approaches which can treat the complete ion-target interaction in a nonperturbative way. 
The here outlined $\mathrm{BC}$ model for the cooling force on a single ion has been compared with classical trajectory Monte Carlo (CTMC) numerical simulations and the simple empirical ansatz (50) proposed by Parkhomchuk. It has been shown that there is a quite good overall qualitative and in most cases also a good quantitative agreement with the CTMC results with respect to the parallel cooling force (18). A similar good qualitative agreement has been observed for the transverse force $F_{\perp}$ (19) but the quantitative agreement with CTMC is here less distinct than for $F_{\|}$. In any case, however, the perturbative BC model and the nonperturbative CTMC based on the full equations of motion in the presence of a magnetic field exhibit a much more intricate structure as provided by the empirical ansatz (50). In a further step we also compared the theoretical cooling force (54), after averaging over the ion distribution function, with the experiments performed at the ESR at GSI [28-30]. The overall agreement of Eq. (54) with the experimental cooling forces is rather good. Unfortunately, a comparison of the averaged cooling force as extracted from the experiments is only little suited for a distinct test of the accuracy of the considered model. By demonstrating the quite involved structure and character of the BC cooling force $\mathbf{F}\left(\mathbf{v}_{i}\right)$ on a single ion we showed, however, that the good agreement with the experimental data cannot simply be considered as accidental. The remaining deviations of Eq. (54) from the ESR data at medium velocities, which can be seen in Fig. 8, are therefore essentially ascribed to the deviations of the model distribution function (52) from the experimental distribution of the ion beam which is not known precisely.

As the main goal of this paper we suggest a more advanced analytical model for calculations of the cooling force which is appropriate for modeling many experimental situations with moderate or strong magnetic guiding fields. The resulting cooling forces $\mathbf{F}\left(\mathbf{v}_{i}\right)$ and $\mathcal{F}(u)$ can, for instance, also be tabulated in a suitable manner to be used as input for simulations of electron cooling using the BETACOOL package $[44,45]$. In addition, further improvement might be achieved by performing the average involved in Eq. (51) numerically with recorded ion beam distributions or analytically using other ion distributions like e.g. the parabolic distribution function as it occurs in CELSIUS [40-42]. Systematic comparisons for different distribution functions and other experiments on electron cooling as well as with CTMC simulations are in progress and will be reported elsewhere.

\section{ACKNOWLEDGMENTS}

One of the authors, H.B. N., is grateful for the support of the Alexander von Humboldt Foundation, Germany. This work was supported by the Bundesministerium für Bildung und Forschung (BMBF) under Contract No. 06ER9064.

\section{APPENDIX A: SOME CONSEQUENCES OF THE COULOMB DIVERGENCY}

As was shown by Parkhomchuk [33] in the $B \rightarrow \infty$ limit and at high velocities, one gets asymptotic expressions for the cooling forces which essentially differ from Eqs. (29)-(32). Here we will briefly show that this is a consequence of the bare Coulomb interaction and the related Coulomb logarithm $\mathcal{U}_{\mathrm{C}}$ used in previous treatments (see, e.g., Refs. $[9,33,40]$ ). As has been argued in Ref. [23] an expression similar to the second-order force (10) strongly depends on the order of the integrations for any singular potential, in particular for $U=U_{\mathrm{C}}$. Such an ambiguity does not arise for any regularized potential and, for instance, Eqs. (21) and (27) are finite. Assuming a finite range of the potential in Eq. (10) we have performed first an integration with respect to the impact parameters $\mathbf{s}$ in whole two-dimensional space. Now let us derive the cooling force (27) first performing the $t$ integration, i.e. changing the order of the $\mathbf{s}$ and $t$ integrations. The calculations are straightforward. Using the trajectory corrections in the presence of an infinitely strong magnetic field derived in Ref. [23], one obtains

$$
\begin{aligned}
F_{\|}\left(\mathbf{v}_{i}\right)= & \frac{2 \pi Z^{2} e^{4} n_{e}}{m} \int\left(2 \mathcal{T}_{1}+\mathcal{T}_{2}\right) \frac{v_{i \perp}^{2} v_{r \|}}{v_{r}^{5}} f\left(\mathbf{v}_{e}\right) d \mathbf{v}_{e}, \\
F_{\perp}\left(\mathbf{v}_{i}\right)= & -\frac{2 \pi Z^{2} k^{4} n_{e}}{m} \int\left[\left(v_{i \perp}^{2}-v_{r \|}^{2}\right) \mathcal{T}_{1}-v_{r \|}^{2} \mathcal{T}_{2}\right] \\
& \times \frac{v_{i \perp}}{v_{r}^{5}} f\left(\mathbf{v}_{e}\right) d \mathbf{v}_{e},
\end{aligned}
$$

where $v_{r \|}=v_{e \|}-v_{i \|}$, and the functions $T_{\nu \mu}(s)$ and quantities $\mathcal{T}_{1}$ and $\mathcal{T}_{2}$ have been introduced in Ref. [23],

$$
\begin{gathered}
\mathcal{T}_{1}=\int_{0}^{\infty} T_{12}^{2}(s) s d s, \quad \mathcal{T}_{2}=\int_{0}^{\infty} T_{03}(s) T_{01}(s) s d s, \\
T_{\nu \mu}(s)=\frac{(2 \pi)^{2}}{2} \int_{0}^{\infty} U(k) J_{\nu}(k s) k^{\mu} d k .
\end{gathered}
$$

In Ref. [23] we have shown that $\mathcal{T}_{1}^{\mathrm{R}}=\mathcal{T}_{2}^{\mathrm{R}}=\mathcal{U}$ for any regularized interaction potential, where $\mathcal{U}$ is given by Eq. (24). Thus, inserting these values of the coefficients $\mathcal{T}_{1}^{\mathrm{R}}$ and $\mathcal{T}_{2}^{\mathrm{R}}$ into Eqs. (A1) and (A2) yields Eqs. (29) and (30), respectively. The situation is different for any unregularized potential, as, for instance, the Debye-like interaction potential $U(k)=U_{\mathrm{D}}(k)$ introduced in Sec. II. For this potential $T_{12}^{\mathrm{D}}(s)=(1 / \lambda) K_{1}(s / \lambda), \quad T_{03}^{\mathrm{D}}(s)=(1 / s) \delta(s)-$ $\left(1 / \lambda^{2}\right) K_{0}(s / \lambda), \quad$ and $\quad T_{01}^{\mathrm{D}}(s)=K_{0}(s / \lambda) \quad$ (see, e.g., Ref. [23] for details), where $K_{n}(z)$ (with $n=0,1$ ) are the modified Bessel functions, and $\lambda$ is the screening length. Transition of these functions to the bare Coulomb case is performed by taking the limit $\lambda \rightarrow \infty$. Then $T_{12}^{\mathrm{C}}(s)=1 / s$ and $T_{01}^{\mathrm{C}}(s) T_{03}^{\mathrm{C}}(s) \rightarrow 0$ in this limit and for any nonzero value of $s>0$. Thus, in Eqs. (A1) and (A2) it can be assumed $\mathcal{T}_{2}^{\mathrm{C}}=0$ while inserting $T_{12}^{\mathrm{C}}(s)$ into Eq. (A3) 
and introducing the upper and lower cutoffs yields $\mathcal{T}_{1}^{\mathrm{C}}=$ $U_{\mathrm{C}}=\ln \left(r_{\max } / r_{\min }\right)$. It is easy to see that Eqs. (A1) and (A2) with $\mathcal{T}_{1}^{\mathrm{C}}$ and $\mathcal{T}_{2}^{\mathrm{C}}=0$ completely agree with the result reported by Parkhomchuk in Ref. [33]. However, it should be emphasized that while the integrand in the coefficient $\mathcal{T}_{2}^{\mathrm{C}}$ tends to zero for a bare Coulomb interaction the $s$ integration of this integrand (i.e. the coefficient $\mathcal{T}_{2}^{\mathrm{C}}$ ) remains singular. This is easily proved by inserting $T_{01}^{\mathrm{D}}(s)$ and $T_{03}^{\mathrm{D}}(s)$ into Eq. (A3). After changing the integration variable the resulting coefficient $\mathcal{T}_{2}^{\mathrm{D}}$ is both independent of the screening length $\lambda$ and diverges logarithmically at small $s$. Consequently, we conclude that for any unregularized potential the coefficient $\mathcal{T}_{2}$ is of the same order as $\mathcal{T}_{1}$ both diverging logarithmically at small $s$ (and possibly at large $s$ ) and the term proportional to $\mathcal{T}_{2}$ cannot be simply neglected in Eqs. (A1) and (A2) as, for instance, in Ref. [33].

\section{APPENDIX B: ADJUSTMENT OF THE EFFECTIVE INTERACTION}

Our results, Eqs. (18), (19), and (54), were derived by using the screened interaction $U_{\mathrm{R}}(r)$. As already mentioned, the use and the modeling of such an effective two-body interaction is a major, but indispensable approximation for a BC treatment where the full ion-target interaction is replaced by an accumulation of isolated ionelectron collisions. The replacement of the complicated real nonspherically symmetric potential, like the wakefields as shown and discussed in Ref. [46], with a spherically symmetric one is, however, well motivated by earlier studies on a BC treatment at vanishing magnetic field, see Refs. [35-37]. There it was shown by comparison with 3D self-consistent PIC simulations that the drag force from the real nonsymmetric potential induced by the moving ion can be well approximated by an $\mathrm{BC}$ treatment employing a symmetric Debye-like potential with an effective velocitydependent screening length $\lambda\left(v_{i}\right)$. In these studies also a recipe was given on how to derive the explicit form of $\lambda\left(v_{i}\right)$, which turned out to be not too much different from a dynamic screening length of the simple form $\lambda\left(v_{i \|}\right)=$ $\lambda_{\mathrm{st}}\left[1+\left(v_{i \|} / v_{s}\right)^{2}\right]^{1 / 2}$. Here $\lambda_{\mathrm{st}}=v_{s} / \omega_{p}$ is the statical screening length at $v_{i \|}=0, \omega_{p}$ is the electron plasma frequency, and $v_{s}$ is a characteristic thermal velocity which depends on the temperature anisotropy of the electron beam and the guiding magnetic field. Although no systematic studies about the use of such an effective interaction with a screening length $\lambda\left(v_{i}\right)$ have been made for ion stopping in a magnetized electron plasma, the replacement of the real interaction by a velocity-dependent spherical one should be a reasonable approximation also in this case. The introduced dynamical screening length $\lambda\left(v_{i \|}\right)$ also implies the assumption of a weak perturbation of the electrons by the ion and linear screening where the screening length is independent of the ion charge $Z e$, which coincide with the regimes of perturbative $\mathrm{BC}$, see, e.g., Ref. [36]. Therefore we do not consider here possible nonlinear screening effects. Supposing linear screening there remains the appropriate choice of the thermal velocity $v_{s}$, which defines the static screening $\lambda_{\mathrm{st}}=v_{s} / \omega_{p}$ at low velocities, the dynamical one $\lambda\left(v_{i \|}\right)=v_{i \|} / \omega_{p}$ at $v_{i \|} \gg v_{s}$, and the velocity scale on which the transition between static and dynamic screenings takes place.

In principle, the screening length $\lambda_{\text {st }}$ can be calculated within the linear-response theory using the dielectric function of a temperature-anisotropic and magnetized plasma (see, e.g., Ref. [11] and references therein). This approach predicts that (i) the quantity $\lambda_{\text {st }}$ is, in general, strongly anisotropic and depends on the angle $\vartheta$ between radius vector $\mathbf{r}$ and magnetic field $\mathbf{B}$ as well as on the strength of the magnetic field and the temperatures $T_{\|}, T_{\perp}$ of the electron plasma. (ii) At vanishing magnetic field the screening length $\lambda_{\text {st }}$ is approximately given by the longitudinal $\lambda_{\mathrm{D} \|}=v_{\text {th } \|} / \omega_{p}$ and the transverse $\lambda_{\mathrm{D} \perp}=v_{\text {th } \perp} / \omega_{p}$ Debye lengths at $\vartheta=0$ and $\vartheta=\pi / 2$, respectively (see, e.g., Ref. [11]). For an average temperature $\bar{T}=$ $\frac{1}{3}\left(T_{\|}+2 T_{\perp}\right)$ of the electrons with corresponding thermal velocity $\bar{v}_{\text {th }}=(\bar{T} / m)^{1 / 2}$ the static screening can be approximated by taking $\lambda_{\text {st }}=\bar{\lambda}_{\mathrm{D}}=\bar{v}_{\text {th }} / \omega_{p}$, where $\bar{\lambda}_{\mathrm{D}}$ can be considered as an angular averaged screening length. (iii) At infinitely strong magnetic field the screening length is only determined by the longitudinal temperature $T_{\|}$of the electrons, $\lambda_{\mathrm{st}}=\lambda_{\mathrm{D} \|}[11]$.

The dielectric properties of a temperature-anisotropic and magnetized plasma thus suggest to define the thermal velocity $v_{s}$ by an interpolation between $\bar{v}_{\text {th }}$ at $B=0$ and $v_{\text {th\| }}$ at $B \rightarrow \infty$, which then covers the entire range of the variation of a guiding magnetic field, from the unmagnetized to the strongly magnetized regimes. To this end, we propose here a simple interpolation formula for the characteristic velocity $\boldsymbol{v}_{s}$, given by

$$
v_{s}^{2}=\frac{\bar{v}_{\mathrm{th}}^{2}+\left(\omega_{c} / \omega_{p}\right)^{\mu} v_{\mathrm{th} \|}^{2}}{1+\left(\omega_{c} / \omega_{p}\right)^{\mu}}
$$

and take $\lambda_{\mathrm{st}}=v_{s} / \omega_{p}$ as static screening length. Here $\mu>0$ is some positive numerical factor and the strength of the magnetic field is measured by the quantity $\omega_{c} / \omega_{p}$. From Eq. (B1) it is seen that the transition from $B=0$ to $B=\infty$ regime is faster for larger $\mu$, where we suggest $\mu=2$ for practical applications. But the explicit functional form of this interpolation as well as the choice of $\mu=2$ are, of course, to a certain extent discretionary. We remark, however, that Eq. (B1) is here basically given to complete our present $\mathrm{BC}$ treatment by providing some reasonable recipe of how to determine the required parameters for modeling the effective interaction. The results shown and discussed in Secs. IV and V are obtained by fixing $v_{s}$ to $\bar{v}_{\text {th }}$ for all cases corresponding to $B=0$ and to $v_{s}=v_{\text {th } \|}$ for all examples with $B \geq 0.1 \mathrm{~T}$ 
(where $\omega_{c} \gg \omega_{p}$ for the assumed parameter regimes). These results are therefore not affected by the explicit form and choice of the suggested interpolation (B1).

It should be also mentioned that, depending on the specific conditions in the storage rings, the screening length $\lambda$ has to be replaced by the radius $r_{0}$ of the electron beam if $r_{0}<\lambda$ [4]. Also the finite time $\tau_{f}$ of flight of the beam through the cooling section may decrease the upper cutoff if $\tau_{f}<\omega_{p}^{-1}$ [47]. However, the first issue is not important for our present comparisons with experimental data [28-30]. The radius of the electron beam and the averaged screening length in these experiments are about $r_{0} \simeq 25 \mathrm{~mm}$ and $\bar{\lambda}_{\mathrm{D}} \simeq 2 \mathrm{~mm}$, respectively, and thus $r_{0} \gg$ $\bar{\lambda}_{\mathrm{D}}$ [29]. The time $\tau_{f}$ for the ESR experimental conditions is unfortunately not significantly larger than $\omega_{p}^{-1}$ [29]. Thus, the stationary picture we use is just applicable but the finite time $\tau_{f}$ is an additional source of uncertainty for the comparison of the present theory and experiment which needs further attention.

Next we specify the parameter $\lambda$ which is a measure of the softening of the interaction potential at short distances. As we discussed in the preceding sections, the regularization of the potential (17) guarantees the existence of the $s$ integrations, but there remains the problem of treating accurately hard collisions. For a perturbative treatment the change in relative velocity of the particles must be small compared to $v_{r}$ and this condition is increasingly difficult to fulfill in the regime $v_{r} \rightarrow 0$. This suggests to enhance the softening of the potential near the origin the smaller $v_{r}$ is. Within the present perturbative treatment, we employ a dynamical regularization parameter $\lambda\left(v_{i \|}\right)$ $[24,25]$, where $\lambda^{2}\left(v_{i \|}\right)=C b_{0}^{2}\left(v_{i \|}\right)+\lambda_{0}^{2}$ and $b_{0}\left(v_{i \|}\right)=$ $|Z| e^{2} / m\left[v_{i \|}^{2}+\left\langle v_{i \perp}^{2}\right\rangle+v_{s}^{2}\right],\left\langle v_{i \perp}^{2}\right\rangle$ is the average of $v_{i \perp}^{2}$ over the ion distribution function (52). This average is $\left\langle v_{i \perp}^{2}\right\rangle=v_{i \perp}^{2}$ in the case of single ion considered in
Secs. II, III, and IV and $\left\langle v_{i \perp}^{2}\right\rangle=2 \sigma_{\perp}^{2}$ in the case of ion beam considered in Sec. V. Here $b_{0}$ is the averaged distance of closest approach of two charged particles in the absence of a magnetic field and $\lambda_{0}$ is some free parameter. In addition we also introduced $C \simeq 0.292$ in $\lambda\left(v_{i \|}\right)$. In Refs. [24,25] this parameter is deduced from the comparison of the second-order scattering cross sections with an exact asymptotic expression derived in Ref. [48] for the Yukawa-type (i.e., with $\lambda \rightarrow 0$ ) interaction potential. As we have shown in Refs. [24,25] employing the dynamical parameter $\lambda\left(v_{i \|}\right)$ the second-order cross sections for electron-electron and electron-ion collisions excellently agree with CTMC simulations at high velocities. Also the free parameter $\lambda_{0}$ is chosen such that $\lambda_{0} \ll b_{0}(0)$, where $b_{0}(0)$ is the distance $b_{0}\left(v_{i \|}\right)$ at $v_{i \|}=0$. From the definition of $\lambda\left(v_{i \|}\right)$ it can be directly inferred that $\lambda_{0}$ does not play any role at low velocities while it somewhat affects the size of the cooling force at high velocities when $b_{0}\left(v_{i \|}\right) \lesssim \lambda_{0}$. More details on the parameter $\lambda_{0}$ and its influence on the cooling force are discussed in Appendix C.

Our extensive numerical calculations indicated that the employed regularization parameter $\lambda\left(v_{i \|}\right)$ provides a qualitatively quite satisfactory description, although the secondorder forces $F_{\| ; \perp}$ on a single ion are at small $v_{i \perp}$, in general, quite sensitive to variations of $\lambda\left(v_{i \|}\right)$. This sensitivity is larger for highly charged ions (like, e.g., $\mathrm{Xe}^{54+}$ ) and in the domain of $v_{i \|}$ where $F_{\|}$gets its minimum (see, e.g., the deep minima in Figs. 5 and 6, left panels). An example of this sensitivity is the formation of the unphysical sawtooth structure in the minimum of the parallel force shown in Fig. 6 (solid lines). Here the regularization parameter $\lambda\left(v_{i \|}\right)$ is no longer capable to capture sufficiently accurately the underlying physics.

Finally, we also illustrate in Fig. 9 the features of the Coulomb logarithm $\mathcal{U}_{\mathrm{R}}=\Lambda(x)$ given by Eq. (25) and the
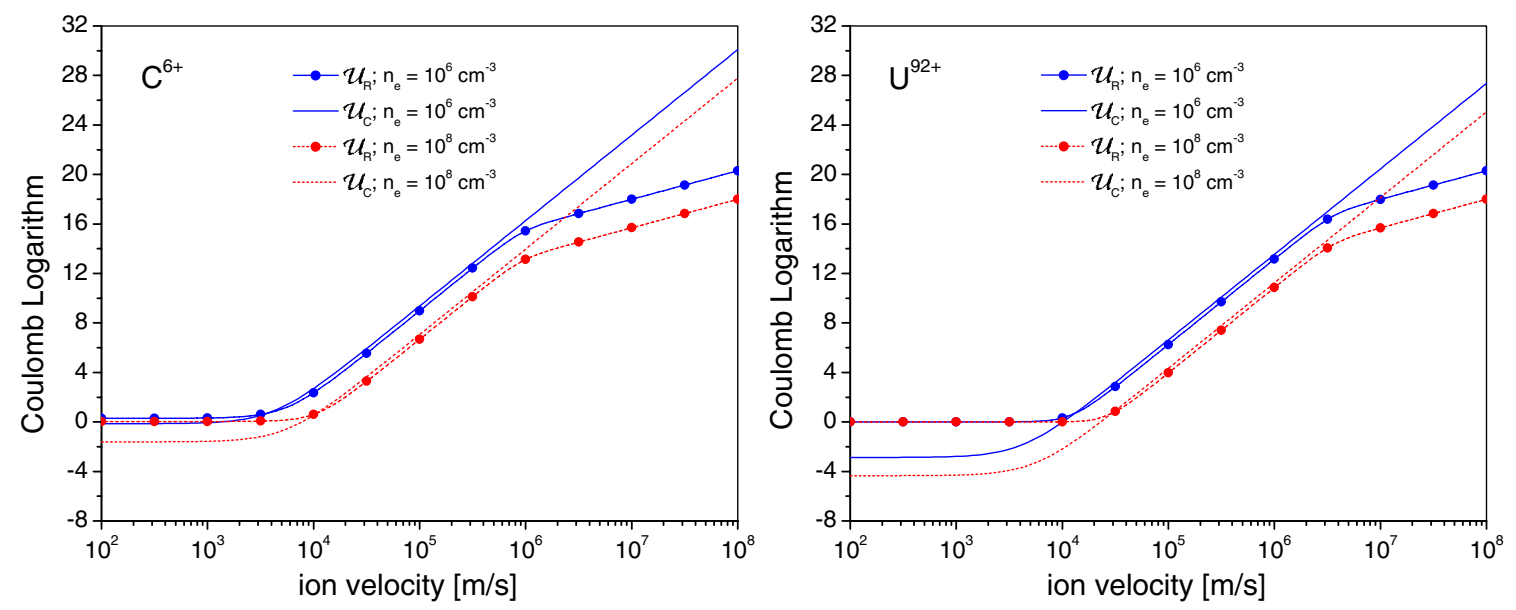

FIG. 9. Regularized $\Lambda(x)$ (the lines with symbols) given by Eq. (25) and standard $\mathcal{U}_{\mathrm{C}}$ (the lines without symbols) Coulomb logarithms for $\mathrm{C}^{6+}$ (left panel) and $\mathrm{U}^{92+}$ (right panel) fully stripped ions as a function of $v_{i \|}$ (in $\mathrm{m} / \mathrm{s}$ ). The Coulomb logarithms are calculated for $\lambda_{0}=10^{-9} \mathrm{~m}, v_{i \perp}=0, B=0.1 \mathrm{~T}$ and for $T_{\perp}=0.11 \mathrm{eV}, T_{\|}=0.1 \mathrm{meV}, n_{e}=10^{6} \mathrm{~cm}^{-3}$ (solid lines), and $n_{e}=10^{8} \mathrm{~cm}-3$ (dotted lines). 
standard one $\mathcal{U}_{\mathrm{C}}=\ln \left(r_{\max } / r_{\min }\right)$ for $B=0.1 \mathrm{~T}$ and for different charge state $Z$ of the ions and temperatures and densities of the electron beam close to the typical values of the experiments at the ESR storage ring [28-30] and many other cooling experiments. For $\mathcal{U}_{\mathrm{C}}$ we take $r_{\max }=\lambda\left(v_{i \|}\right)$ and $r_{\min }=b_{0}\left(v_{i \|}\right)$. The velocity-dependent lengths $\lambda\left(v_{i \|}\right)$ and $b_{0}\left(v_{i \|}\right)$ have been defined and discussed above. These lengths also fix the quantity $x\left(v_{i \|}\right)=1+\lambda\left(v_{i \|}\right) / \lambda\left(v_{i \|}\right)$ used for $\mathcal{U}_{\mathrm{R}}$. As can be seen from Fig. 9, at intermediate velocities the Coulomb logarithm $\mathcal{U}_{\mathrm{R}}=\Lambda(x)$ basically shows the same behavior and features as $\mathcal{U}_{\mathrm{C}}$, but results here in a somewhat smaller cooling force. Deviations are more pronounced at high velocities when the distance of the closest approach become comparable or smaller than the regularization parameter $\lambda_{0}, b_{0}\left(v_{i \|}\right) \lesssim \lambda_{0}$. It is clear that decreasing the parameter $\lambda_{0}$ will result in a shift of the deviation domain shown in Fig. 9 towards higher velocities. We like to emphasize, however, that the large deviations between both Coulomb logarithms shown in Fig. 9 fall in the velocity domain where the resulting cooling forces are usually very small (see, e.g., the examples shown in Figs. 1-8). Finally at small velocities the standard Coulomb logarithm becomes negative (i.e. $r_{\max }<r_{\min }$ ) which indicates the violation of the perturbative approach, and is more pronounced either at higher densities $n_{e}$ or larger ion charge; see Fig. 9.

\section{APPENDIX C: COOLING FORCE VERSUS THE PARAMETER $\boldsymbol{\lambda}_{\mathbf{0}}$}

Finally, we briefly investigate the influence of the choice of different values of the free parameter $\lambda_{0}$ on the cooling

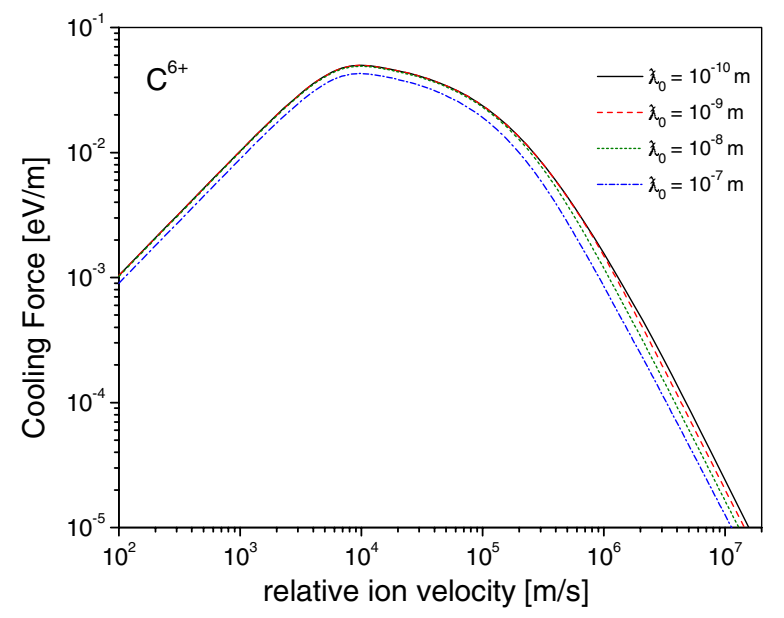

FIG. 10. Longitudinal cooling force (in $\mathrm{eV} / \mathrm{m}$ ) for the $\mathrm{C}^{6+}$ ion as a function of the relative ion velocity (in $\mathrm{m} / \mathrm{s}$ ). The theoretical cooling force (54) is calculated for an electron beam with $n_{e}=$ $10^{6} \mathrm{~cm}^{-3}, T_{\perp}=0.11 \mathrm{eV}$, and $T_{\|}=0.1 \mathrm{meV}$ in a magnetic field of $B=0.1 \mathrm{~T}$ for $\lambda_{0}=10^{-10} \mathrm{~m}$ (solid line), $\lambda_{0}=10^{-9} \mathrm{~m}$ (dashed line), $\lambda_{0}=10^{-8} \mathrm{~m}$ (dotted line), and $\lambda_{0}=10^{-7} \mathrm{~m}$ (dash-dotted line). The ion beam is characterized by the distribution $\sigma_{\|}=0$, $\sigma_{\perp}=3.5 v_{i \perp}^{*},\left\langle\theta_{i}\right\rangle=0.2 \mathrm{mrad}$ (see Sec. V B for details). force (54). As mentioned in Appendix B, this parameter is chosen such that $\lambda_{0} \ll b_{0}(0)$ and therefore does not play any role at low velocities. It adjusts, however, the cooling force in the high-velocity regime when $b_{0}\left(\tilde{v}_{i \|}\right) \lesssim \lambda_{0}$. Only in this high-velocity limit the parameter $\lambda_{0}$ directly affects (within logarithmic accuracy) the perturbative cooling force via the generalized Coulomb logarithm $\Lambda(x)$ determined by Eq. (25). Thereby $\Lambda(x)$ depends on the ion beam velocity $\tilde{v}_{i \|}$ and behaves at high velocities as $\Lambda(x) \simeq \ln x-$ $1 \simeq \ln \left(\tilde{v}_{i \|} / \omega_{p} \lambda_{0}\right)-1$. This velocity dependence of $\Lambda(x)$ must be taken into account when considering the asymptotic expressions (55) and (56).

For the curves plotted in Fig. 10 we evaluated the cooling force expression (54) for an $\mathrm{C}^{6+}$ ion varying the regularization parameter from $\lambda_{0}=10^{-10} \mathrm{~m}$ (solid line) to $\lambda_{0}=10^{-7} \mathrm{~m}$ (dash-dotted line). All other parameters remain fixed and are essentially the same as in Fig. 8. For $\lambda_{0} \leq 10^{-8} \mathrm{~m}$ the cooling force is (weakly) sensitive to a variation of $\lambda_{0}$, but as expected, only in the high-velocity domain. At the larger $\lambda_{0}=10^{-7} \mathrm{~m}$, where the parameter $\lambda_{0}$ becomes comparable to the static collision diameter, $\lambda_{0} \simeq b_{0}(0)$, the cooling force shows some sensitivity to $\lambda_{0}$ also at low velocities (dash-dotted line) resulting in an overall decrease of the force. But for the higher charged ions, as considered in Sec. V B, the collision distance $b_{0}$ is larger and the sensitivity of the cooling force to $\lambda_{0}$ thus starts at even larger $\lambda_{0}$.

[1] G. I. Budker, At. Energ. 22, 346 (1967) [Sov. At. Energy 22, 438 (1967)].

[2] A. H. Sørensen and E. Bonderup, Nucl. Instrum. Methods 215, 27 (1983).

[3] H. Poth, Phys. Rep. 196, 135 (1990).

[4] I. N. Meshkov, Phys. Part. Nucl. 25, 631 (1994).

[5] L. I. Men'shikov, Phys. Usp. 51, 645 (2008).

[6] M. Amoretti et al., Nature (London) 419, 456 (2002).

[7] G. Gabrielse et al., Phys. Rev. Lett. 89, 213401 (2002).

[8] W. Quint et al., Hyperfine Interact. 132, 457 (2001).

[9] Ya. S. Derbenev and A. N. Skrinsky, Part. Accel. 8, 235 (1978).

[10] M. Walter, Ph.D. thesis, University of Erlangen, 2002.

[11] H. B. Nersisyan, M. Walter, and G. Zwicknagel, Phys. Rev. E 61, 7022 (2000).

[12] M. Walter, C. Toepffer, and G. Zwicknagel, Nucl. Instrum. Methods Phys. Res., Sect. B 168, 347 (2000).

[13] B. Möllers, C. Toepffer, M. Walter, G. Zwicknagel, C. Carli, and H. B. Nersisyan, Nucl. Instrum. Methods Phys. Res., Sect. A 532, 279 (2004).

[14] G. Zwicknagel, habilitation treatise, University of Erlangen, $2000 \quad$ [http://www.opus.ub.uni-erlangen.de/ opus/volltexte/2008/913/].

[15] G. Zwicknagel, in Non-Neutral Plasma Physics III, edited by J. B. Bollinger, R. L. Spencer, and R. C. Davidson, AIP Conf. Proc. No. 498 (AIP, New York, 1999), p. 469.

[16] G. Zwicknagel and C. Toepffer, in Non-Neutral Plasma Physics IV, edited by F. Anderegg, L. Schweikhard, and 
C. F. Driscoll, AIP Conf. Proc. No. 606 (AIP, New York, 2002), p. 499.

[17] G. Zwicknagel, in Beam Cooling and Related Topics, edited by S. Nagaitsev and R. J. Pasquinelli, AIP Conf. Proc. No. 821 (2006), p. 513.

[18] G. Zwicknagel, Trapped Charged Particles and Fundamental Interactions, Lecture Notes in Physics Vol. 749, edited by K. Blaum and F. Herfurth (SpringerVerlag, Berlin, 2008), pp. 69-96.

[19] C. Toepffer, Phys. Rev. A 66, 022714 (2002).

[20] B. Möllers, M. Walter, G. Zwicknagel, C. Carli, and C. Toepffer, Nucl. Instrum. Methods Phys. Res., Sect. B 207, 462 (2003).

[21] H. B. Nersisyan, Nucl. Instrum. Methods Phys. Res., Sect. B 205, 276 (2003).

[22] H. B. Nersisyan, G. Zwicknagel, and C. Toepffer, Phys. Rev. E 67, 026411 (2003).

[23] H.B. Nersisyan, C. Toepffer, and G. Zwicknagel, Interactions Between Charged Particles in a Magnetic Field: A Theoretical Approach to Ion Stopping in Magnetized Plasmas (Springer, Heidelberg, 2007).

[24] H. B. Nersisyan and G. Zwicknagel, Phys. Rev. E 79, 066405 (2009).

[25] H. B. Nersisyan and G. Zwicknagel, Phys. Plasmas 17, 082314 (2010).

[26] G. Kelbg, Ann. Phys. (Berlin) 467, 219 (1963).

[27] C. Deutsch, Phys. Lett. A 60, 317 (1977).

[28] T. Winkler et al., Hyperfine Interact. 99, 277 (1996).

[29] T. Winkler, Ph.D. thesis, University Heidelberg, 1996.

[30] T. Winkler, K. Beckert, F. Bosch, H. Eickhoff, B. Franzke, F. Nolden, H. Reich, B. Schlitt, and M. Steck, Nucl. Instrum. Methods Phys. Res., Sect. A 391, 12 (1997).

[31] V. V. Parkhomchuk, Nucl. Instrum. Methods Phys. Res., Sect. A 441, 9 (2000).

[32] V. V. Parkhomchuk and A. N. Skrinskii, Phys. Usp. 43, 433 (2000).

[33] V. V. Parkhomchuk, in Proceedings of the Workshop on Electron Cooling and Related Applications,
ECOOL84, 1984, edited by H. Poth (KfK, Karlsruhe, 1985), p. 71.

[34] A. I. Akhiezer, I. A. Akhiezer, R. V. Polovin, A. G. Sitenko, and K. N. Stepanov, Plasma Electrodynamics (Pergamon, Oxford, 1975), Vol. 1, 1st ed.

[35] G. Zwicknagel, Laser Part. Beams 27, 399 (2009).

[36] G. Zwicknagel, Nucl. Instrum. Methods Phys. Res., Sect. B 197, 22 (2002).

[37] G. Zwicknagel, C. Toepffer, and P.-G. Reinhard, Phys. Rep. 309, 117 (1999); 314, 671(E) (1999).

[38] I. S. Gradshteyn and I. M. Ryzhik, Tables of Integrals, Series and Products (Academic, New York, 1980) 2nd ed.

[39] D. V. Pestrikov, Nucl. Instrum. Methods Phys. Res., Sect. A 554, 13 (2005).

[40] A. V. Fedotov, D. Bruhwiler, A. Sidorin, D. Abell, I. Ben-Zvi, R. Busby, J. Cary, and V. Litvinenko, Phys. Rev. ST Accel. Beams 9, 074401 (2006).

[41] A. V. Fedotov, B. Gålnander, V. Litvinenko, T. Lofnes, A. Sidorin, A. Smirnov, and V. Ziemann, Phys. Rev. E 73, 066503 (2006).

[42] A. V. Fedotov et al., in , Beam Cooling and Related Topics, edited by R. J. Pasquinelli, AIP Conf. Proc. No. 821 (AIP, New York, 2006), p. 265.

[43] M. Beutelspacher, M. Grieser, K. Noda, and T. Shirai, in Proceedings of the Workshop on Ion Beam Cooling: Toward the Crystalline Beam, Kyoto, Japan, 2001, edited by A. Noda and T. Shirai (World Scientific, Singapore, 2002), pp. 93-128.

[44] A. Yu. Lavrentev and I.N. Meshkov, JINR Report No. E9-95-317.

[45] N. Madsen, Report No. CERN/PS/DI/Note 99-20, AD Note 053 (1999).

[46] T. Peter and J. Meyer-ter-Vehn, Phys. Rev. A 43, 1998 (1991).

[47] C. Seele, G. Zwicknagel, C. Toepffer, and P.-G. Reinhard, Phys. Rev. E 57, 3368 (1998).

[48] H. Hahn, E. A. Mason, and F. J. Smith, Phys. Fluids 14, 278 (1971). 